\title{
STRUCTURE THEORY AND STABLE RANK FOR C*-ALGEBRAS OF FINITE HIGHER-RANK GRAPHS
}

\author{
DAVID PASK, ADAM SIERAKOWSKI, AND AIDAN SIMS
}

\begin{abstract}
We study the structure and compute the stable rank of $C^{*}$-algebras of finite higher-rank graphs. We completely determine the stable rank of the $C^{*}$ algebra when the $k$-graph either contains no cycle with an entrance, or is cofinal. We also determine exactly which finite, locally convex $k$-graphs yield unital stably finite $C^{*}$-algebras. We give several examples to illustrate our results.
\end{abstract}

\section{INTRODUCTION}

Stable rank is a non-commutative analogue of topological dimension for $C^{*}$ algebras introduced by Rieffel in the early 1980s [34], and widely used and studied ever since (see, for example [33, 30, 10, 46, 18, 11, 36, 24, 12, 4, 15, 44]). The condition of having stable rank 1 , meaning that the invertible elements are dense in the $C^{*}$-algebra, has attracted significant attention, in part due to its relevance to classification of $C^{*}$-algebras. Specifically, all separable, simple, unital, nuclear, $\mathcal{Z}$-stable $C^{*}$-algebras in the UCT class are classified by their Elliott invariant $[6,13,19,29,40]$, and stable rank distinguishes two key cases: the stably finite $C^{*}$-algebras in this class have stable rank 1 [36, Theorem 6.7], while the remainder are Kirchberg algebras with stable rank infinity [34, Proposition 6.5]. It follows that a simple $C^{*}$-algebra whose stable rank is finite but not equal to 1 does not belong to the class of $C^{*}$ algebras classified by their Elliott invariants.

Higher rank graphs (or $k$-graphs) $\Lambda$ are generalisations of directed graphs. They give rise to an important class of $C^{*}$-algebras $C^{*}(\Lambda)$ due to their simultaneous concreteness of presentation and diversity of structure $[8,22,26]$. They provide good test cases for general theory [5, 43] and have found unexpected applications in general $C^{*}$-algebra theory. For example, the first proof that Kirchberg algebras in the UCT class have nuclear dimension 1 proceeded by realising them as direct limits of 2-graph $C^{*}$-algebras [39]. Nevertheless, and despite their deceptively elementary presentation in terms of generators and relations, $k$-graph $C^{*}$-algebras in general remain somewhat mysterious - for example it remains an unanswered question whether all simple $k$-graph $C^{*}$-algebras are $\mathcal{Z}$-stable and hence classifiable. This led us to investigate their stable rank. In this paper we shed some light on how to compute the stable rank of $k$-graph $C^{*}$-algebras; though unfortunately, the simple $C^{*}$-algebras to which our results apply all have stable rank either 1 or $\infty$, so we obtain no new information about $\mathcal{Z}$-stability or classifiability.

Date: September 8, 2021.

2010 Mathematics Subject Classification. 46L05.

Key words and phrases. Higher-rank graph; stable rank; $C^{*}$-algebra; operator algebra.

This research was supported by Australian Research Council grant DP180100595. 
This paper focuses on unital $k$-graph $C^{*}$-algebras. For $k=1$, i.e., for directed graph $C^{*}$-algebras (unital or not), a complete characterisation of stable rank has been obtained $[9,18,17]$. In this paper our main contribution is for $k \geq 2$, a characterisation of stable rank for $C^{*}$-algebras associated to

(1) finite $k$-graphs that have no cycle with an entrance, and

(2) finite $k$-graphs that are cofinal.

In the first case (1) we prove that such $k$-graphs are precisely the ones for which the associated $C^{*}$-algebra is stably finite. Partial results on how to characterise stably finite $k$-graph $C^{*}$-algebras have appeared in the past, see [7, 28, 41]. It turns out that in the unital situation, all such $C^{*}$-algebras are direct sums of matrix algebras over commutative tori of dimension at most $k$; the precise dimensions of the tori is determined by the degrees of certain cycles (called initial cycles) in the $k$-graph. Their $C^{*}$-algebraic structure is therefore independent of the factorisation property that determines how the one-dimensional subgraphs of a $k$-graph fit together to give it its $k$-dimensional nature.

We also settle the second case (2) where the $k$-graphs are cofinal using our characterisation of stable finiteness in combination with a technical argument on the von Neumann equivalence of (direct sums of) vertex projections. We initially obtained this result for selected 2-graphs using Python.

We now give a brief outline of the paper; Figure 1 may also help the reader to navigate. In Section 1 we introduce terminology, including the notion (and examples) of an initial cycle. In Section 2 we consider the stably finite case. In Proposition 2.7 we prove that stable finiteness of $C^{*}(\Lambda)$ is equivalent to the condition that no cycle in the $k$-graph $\Lambda$ has an entrance. In Theorem 2.5 we characterise the structure of $C^{*}(\Lambda)$ in the stably finite case and compute the stable rank of such algebras in Corollary 2.9. In Section 3 we characterise which $k$-graphs yield $C^{*}$-algebras with stable rank 1 (Theorem 3.1 and Corollary 3.4) and show how the dimension of the tori that form the components of their spectra can be read off from (the skeleton of) the $k$-graph, see Proposition 3.3.

In Section 4 we look at $k$-graphs which are cofinal. Firstly, in Proposition 4.1 , we study the special case when $C^{*}(\Lambda)$ is simple. Then, in Theorem 4.4 , we compute stable rank when $\Lambda$ is cofinal and contains a cycle with an entrance (so $C^{*}(\Lambda)$ is not stably finite). In Section 5 we illustrate the difficulty in the remaining case where $\Lambda$ is not cofinal and contains a cycle with an entrance by considering 2-vertex 2-graphs with this property. We are able to compute the stable rank exactly for all but three classes of examples, for which the best we can say is that the stable rank is either 2 or 3 .

\section{BACKGROUND}

In this section we recall the definition of stable rank, and the notions of stably finite and purely infinite $C^{*}$-algebras. We also recall the definitions of $k$-graphs and their associated $C^{*}$-algebras. We discuss the path space of a locally convex $k$-graph and describe initial cycles and their periodicity group. The reader familiar with these terms can skim through or skip this section. 


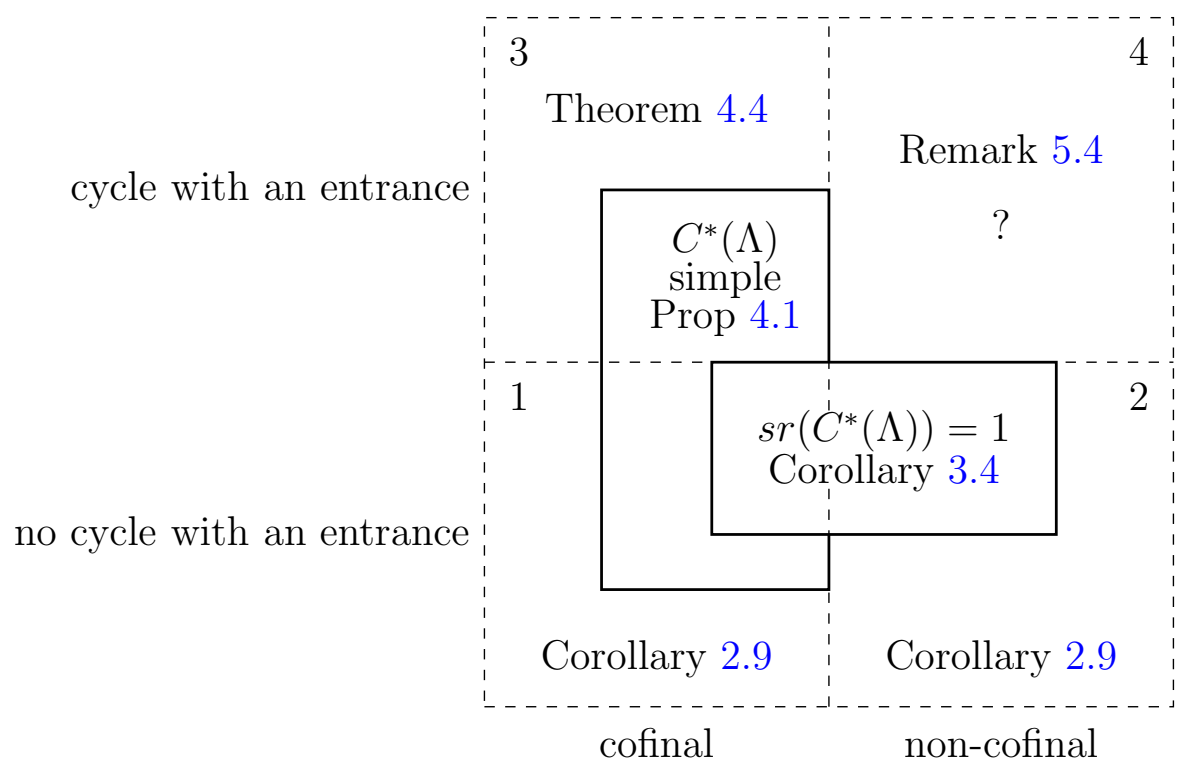

FiguRE 1. Overview of some of our results. The "?" indicates unknown stable rank.

1.1. Stable rank of $C^{*}$-algebras. Let $A$ be a unital $C^{*}$-algebra. Following [2], let

$$
\operatorname{Lg}_{n}(A):=\left\{\left(x_{i}\right)_{i=1}^{n} \in A^{n}: \exists\left(y_{i}\right)_{i=1}^{n} \in A^{n} \text { such that } \sum_{i=1}^{n} y_{i} x_{i}=1\right\} .
$$

The stable rank of $A$, denoted $\operatorname{sr}(A)$, is the smallest $n$ such that $L g_{n}(A)$ is dense in $A^{n}$, or $\infty$ if there is no such $n$. For a $C^{*}$-algebra $A$ without a unit, we define its stable rank to be that of its minimal unitisation $\widetilde{A}$.

A $C^{*}$-algebra $A$ has stable rank one if and only if the set $A^{-1}$ of invertible elements in $A$ is dense in $A$. We will make frequent use of the following key results concerning the stable rank of $C^{*}$-algebras of functions on tori, matrix algebras, stable $C^{*}$ algebras and direct sums later in the paper:

(1) $\operatorname{sr}\left(C\left(\mathbb{T}^{\ell}\right)\right)=\lfloor\ell / 2\rfloor+1$;

(2) $\operatorname{sr}\left(M_{n}(A)\right)=\lceil(\operatorname{sr}(A)-1) / n\rceil+1$;

(3) $\operatorname{sr}(A \otimes \mathcal{K})=1$ if $\operatorname{sr}(A)=1$, and $\operatorname{sr}(A \otimes \mathcal{K})=2$ if $\operatorname{sr}(A) \neq 1$; and

(4) $\operatorname{sr}(A \oplus B)=\max (\operatorname{sr}(A), \operatorname{sr}(B))$.

Stable rank is in general not preserved under Morita equivalence (unless the stable rank is one). For more details see [34].

1.2. Stably finite and purely infinite $C^{*}$-algebras. A projection in a $C^{*}$-algebra is said to be infinite if it is (von Neumann) equivalent to a proper subprojection of itself. If a projection is not infinite it is said to be finite. A unital $C^{*}$-algebra $A$ is said to be finite if its unit is a finite projection, and stably finite if $M_{n}(A)$ is finite for each positive integer $n$ [37, Definition 5.1.1]. We refer to $[7,41]$ for results about stably finite graph $C^{*}$-algebras.

A simple $C^{*}$-algebra $A$ is purely infinite if every nonzero hereditary sub- $C^{*}$-algebra of $A$ contains an infinite projection. For the definition when $A$ is non-simple we refer the reader to [20]. 
1.3. Higher rank graphs. Following [21, 25, 32] we recall some terminology for $k$-graphs. For $k \geq 1$, a $k$-graph is a nonempty, countable, small category equipped with a functor $d: \Lambda \rightarrow \mathbb{N}^{k}$ satisfying the factorisation property: For all $\lambda \in \Lambda$ and $m, n \in \mathbb{N}^{k}$ such that $d(\lambda)=m+n$ there exist unique $\mu, \nu \in \Lambda$ such that $d(\mu)=m$, $d(\nu)=n$, and $\lambda=\mu \nu$. When $d(\lambda)=n$ we say $\lambda$ has degree $n$, and we define $\Lambda^{n}:=d^{-1}(n)$. If $k=1$, then $\Lambda$ is isomorphic to the free category generated by the directed graph with edges $\Lambda^{1}$ and vertices $\Lambda^{0}$. The generators of $\mathbb{N}^{k}$ are denoted $e_{1}, \ldots, e_{k}$, and $n_{i}$ denotes the $i^{\text {th }}$ coordinate of $n \in \mathbb{N}^{k}$. For $m, n \in \mathbb{N}^{k}$, we write $m \leq n$ if $m_{i} \leq n_{i}$ for all $i$, and we write $m \vee n$ for the coordinatewise maximum of $m$ and $n$, and $m \wedge n$ for the coordinatewise minimum of $m$ and $n$.

If $\Lambda$ is a $k$-graph, its vertices are the elements of $\Lambda^{0}$. The factorisation property implies that the vertices are precisely the identity morphisms, and so can be identified with the objects. For each $\lambda \in \Lambda$ the source $s(\lambda)$ is the domain of $\lambda$, and the range $r(\lambda)$ is the codomain of $\lambda$ (strictly speaking, $s(\lambda)$ and $r(\lambda)$ are the identity morphisms associated to the domain and codomain of $\lambda$ ). Given $\lambda, \mu \in \Lambda, n \in \mathbb{N}^{k}$ and $E \subseteq \Lambda$, we define

$$
\begin{aligned}
\lambda E & :=\{\lambda \nu: \nu \in E, r(\nu)=s(\lambda)\}, \\
E \mu & :=\{\nu \mu: \nu \in E, s(\nu)=r(\mu)\}, \\
\Lambda^{\leq n} & :=\left\{\lambda \in \Lambda: d(\lambda) \leq n \text { and } s(\lambda) \Lambda^{e_{i}}=\emptyset \text { whenever } d(\lambda)+e_{i} \leq n\right\} .
\end{aligned}
$$

We say that a $k$-graph $\Lambda$ is row-finite if $\left|v \Lambda^{n}\right|<\infty$ is finite for each $n \in \mathbb{N}^{k}$ and $v \in \Lambda^{0}$, finite if $\left|\Lambda^{n}\right|<\infty$ for all $n \in \mathbb{N}^{k}$, and locally convex if for all distinct $i, j \in\{1, \ldots, k\}$, and all paths $\lambda \in \Lambda^{e_{i}}$ and $\mu \in \Lambda^{e_{j}}$ such that $r(\lambda)=r(\mu)$, the sets $s(\lambda) \Lambda^{e_{j}}$ and $s(\mu) \Lambda^{e_{i}}$ are nonempty.

Standing Assumption. We have two standing assumptions. The first is that all of our $k$-graphs $\Lambda$ are finite. This implies, in particular that they are row-finite. The second is that all of our $k$-graphs $\Lambda$ are locally convex.

A vertex $v$ is called a source if there exist $i \leq k$ such that $v \Lambda^{e_{i}}=\emptyset$. The term cycle, distinct from "generalised cycle" [14], will refer to a path $\lambda \in \Lambda \backslash \Lambda^{0}$ such that $r(\lambda)=s(\lambda)$.

We will occasionally illustrate $k$-graphs as $k$-coloured graphs. We refer to [16] for the details, but in short there is a one-to-one correspondence between $k$-graphs and $k$-coloured graphs together with factorisation rules for bi-coloured paths of length 2 satisfying an associativity condition [16, Equation (3.2)].

1.4. Higher rank graph $C^{*}$-algebras. Let $\Lambda$ be a row-finite, locally convex $k$ graph. Following [32], a Cuntz-Krieger $\Lambda$-family in a $C^{*}$-algebra $B$ is a function $s: \lambda \mapsto s_{\lambda}$ from $\Lambda$ to $B$ such that

(1) $\left\{s_{v}: v \in \Lambda^{0}\right\}$ is a collection of mutually orthogonal projections;

(2) $s_{\mu} s_{\nu}=s_{\mu \nu}$ whenever $s(\mu)=r(\nu)$;

(3) $s_{\lambda}^{*} s_{\lambda}=s_{s(\lambda)}$ for all $\lambda \in \Lambda$; and

(4) $s_{v}=\sum_{\lambda \in v \Lambda \leq n} s_{\lambda} s_{\lambda}^{*}$ for all $v \in \Lambda^{0}$ and $n \in \mathbb{N}^{k}$.

The $C^{*}$-algebra $C^{*}(\Lambda)$ is the universal $C^{*}$-algebra generated by a Cuntz-Krieger $\Lambda$-family. It is unital if and only if $\left|\Lambda^{0}\right|<\infty$, in which case $1=\sum_{v \in \Lambda^{0}} s_{v}$. The universal family in $C^{*}(\Lambda)$ is denoted $\left\{s_{\lambda}: \lambda \in \Lambda\right\}$. 
1.5. The path space of a $k$-graph. Let $\Lambda$ be a $k$-graph. For each path $\lambda \in \Lambda$, and $m \leq n \leq d(\lambda)$, we denote by $\lambda(m, n)$ the unique element of $\Lambda^{n-m}$ such that $\lambda=\lambda^{\prime} \lambda(m, n) \lambda^{\prime \prime}$ for some $\lambda^{\prime}, \lambda^{\prime \prime} \in \Lambda$ with $d\left(\lambda^{\prime}\right)=m$ and $d\left(\lambda^{\prime \prime}\right)=d(\lambda)-n$. We abbreviate $\lambda(m, m)$ by $\lambda(m)$. A $k$-graph morphism between two $k$-graphs is a degree preserving functor.

Following [14], for each $m \in(\mathbb{N} \cup\{\infty\})^{k}$, we define a $k$-graph $\Omega_{k, m}$ by $\Omega_{k, m}=$ $\left\{(p, q) \in \mathbb{N}^{k} \times \mathbb{N}^{k}: p \leq q \leq m\right\}$ with range map $r(p, q)=(p, p)$, source map $s(p, q)=$ $(q, q)$, and degree map $d(p, q)=q-p$. We identify $\Omega_{k, m}^{0}$ with $\left\{p \in \mathbb{N}^{k}: p \leq m\right\}$ via the map $(p, p) \mapsto p$. Given a $k$-graph and $m \in \mathbb{N}^{k}$ there is a bijection from $\Lambda^{m}$ to the set of morphisms $x: \Omega_{k, m} \rightarrow \Lambda$, given by $\lambda \mapsto((p, q) \mapsto \lambda(p, q))$; the inverse is the map $x \mapsto x(0, m)$. Thus, for each $m \in \mathbb{N}^{k}$ we may identify the collection of $k$-graph morphisms from $\Omega_{k, m}$ to $\Lambda$ with $\Lambda^{m}$. We extend this idea beyond $m \in \mathbb{N}^{k}$ as follows: Given $m \in(\mathbb{N} \cup\{\infty\})^{k} \backslash \mathbb{N}^{k}$, we regard each $k$-graph morphism $x: \Omega_{k, m} \rightarrow \Lambda$ as a path of degree $m$ in $\Lambda$ and write $d(x):=m$ and $r(x):=x(0)$; we denote the set of all such paths by $\Lambda^{m}$. We denote by $W_{\Lambda}$ the collection $\bigcup_{m \in(\mathbb{N} \cup\{\infty\})^{k}} \Lambda^{m}$ of all paths in $\Lambda$; our conventions allow us to regard $\Lambda$ as a subset of $W_{\Lambda}$. We call $W_{\Lambda}$ the path space of $\Lambda$. We set

$$
\Lambda^{\leq \infty}=\left\{x \in W_{\Lambda}: x(n) \Lambda^{e_{i}}=\emptyset \text { whenever } n \leq d(x) \text { and } n_{i}=d(x)_{i}\right\}
$$

and for $v \in \Lambda^{0}$, we define $v \Lambda \leq \infty:=\left\{x \in \Lambda^{\leq \infty}: r(x)=v\right\}$. Given a cycle $\tau$, we define $\tau^{\infty}$ (informally written as $\tau^{\infty}:=\tau \tau \tau \ldots$ ) to be the unique element of $W_{\Lambda}$ such that $d\left(\tau^{\infty}\right)_{i}$ is equal to $\infty$ when $d(\tau)_{i}>0$ and equal to 0 when $d(\tau)_{i}=0$, and such that $\left(\tau^{\infty}\right)(n \cdot d(\tau),(n+1) \cdot d(\tau))=\tau$ for all $n \in \mathbb{N}$.

1.6. Initial cycles and their periodicity group. In this section we introduce initial cycles and their associated periodicity group. As we will see in Corollary 2.9 and Theorem 3.1, the periodicity group plays an important role in the characterisation of stable rank.

Let $\lambda$ be a cycle in a row-finite, locally convex $k$-graph $\Lambda$. We say $\lambda$ is a cycle with an entrance if there exists $\tau \in r(\lambda) \Lambda$ such that $d(\tau) \leq d\left(\lambda^{\infty}\right)$ and $\tau \neq \lambda^{\infty}(0, d(\tau))$.

Definition 1.1. ([14]) Let $\Lambda$ be a finite, locally convex $k$-graph that has no cycle with an entrance. We call $\mu \in \Lambda$ an initial cycle if $r(\mu)=s(\mu)$ and if $r(\mu) \Lambda^{e_{i}}=\emptyset$ whenever $d(\mu)_{i}=0$.

Remark 1.2. While the wording of Definition 1.1 differs from that of [14], we will show (in Proposition 2.7) that for any $k$-graph $\Lambda$, a path $\mu \in \Lambda$ is an initial cycle in the sense of Definition 1.1 if and only if it is an initial cycle in the sense of [14].

Remark 1.3. An initial cycle may be trivial, in the sense that it has degree 0 , so it is in fact a vertex. This vertex must then be a source, as for example $w_{4}$ in Figure 5. It is not true that every source is an initial cycle; for example $w_{3}$ in Figure 5 is a source but not an initial cycle.

As in [14], we let $\operatorname{IC}(\Lambda)$ denote the collection of all initial cycles in $\Lambda$; if $\Lambda^{0}$ is finite and $\Lambda$ has no cycle with an entrance, then $\operatorname{IC}(\Lambda)$ is nonempty - see Lemma 2.2. A vertex $v \in \Lambda^{0}$ is said to be on the initial cycle $\mu$ if $v=\mu(p)$ for some $p \leq d(\mu)^{\dagger}$. We

\footnotetext{
${ }^{\dagger}$ This is not the definition in [14, p. 202], but we expect this was the intended definition.
} 

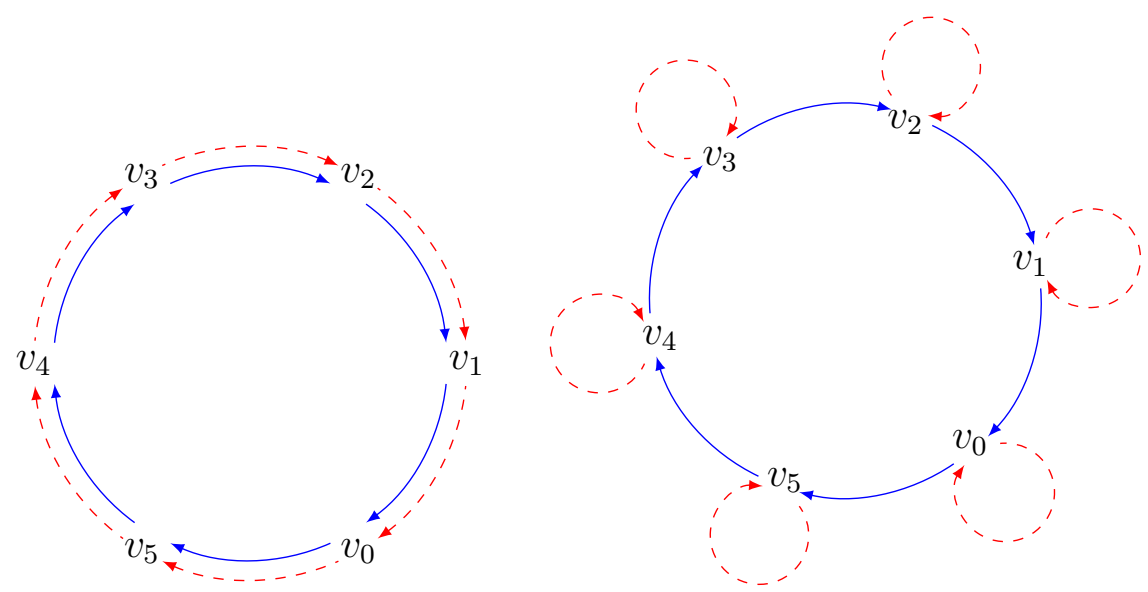

Figure 2. Two 2-graphs $\Lambda_{1}, \Lambda_{2}$, each containing lots of initial cycles, but only one such up to $\sim$-equivalence.

let $\left(\mu^{\infty}\right)^{0}$ denote the collection of all vertices on an initial cycle $\mu$ and let $\sim$ be the equivalence relation on $\operatorname{IC}(\Lambda)$ given by $\mu \sim \nu \Leftrightarrow\left(\mu^{\infty}\right)^{0}=\left(\nu^{\infty}\right)^{0}$.

Remark 1.4. For a finite, locally convex $k$-graph that has no cycle with an entrance, each initial cycle is an "initial segment" in the following sense:

(1) Every path with range on the initial cycle is in the initial cycle, so paths can not "enter" an initial cycle (see Lemma 2.1).

Without the assumption that $\Lambda^{0}$ is finite and $\Lambda$ has no cycle with an entrance property (1) might fail. This is for example the case for the 1-graph with one vertex and two edges representing the Cuntz algebra $\mathcal{O}_{2}$. This suggests that, in general, a different terminology should perhaps be used.

As in [14] we associate a group $G_{\mu}$ to each initial cycle $\mu$. Let $\Lambda$ be a finite, locally convex $k$-graph that has no cycle with an entrance. Let $\mu$ be an initial cycle in $\Lambda$. If $\mu$ is not a vertex, we define

$$
G_{\mu}:=\left\{m-n: n, m \leq d\left(\mu^{\infty}\right), \mu^{\infty}(m)=\mu^{\infty}(n)\right\},
$$

otherwise, we let $G_{\mu}:=\{0\}$.

Definition 1.5. By [14, Lemma 5.8], $G_{\mu}$ is a subgroup of $\mathbb{Z}^{k}$, and hence isomorphic to $\mathbb{Z}^{\ell_{\mu}}$ for some $\ell_{\mu} \in\{0, \ldots, k\}$ : we often refer to $\ell_{\mu}$ as the rank of $G_{\mu}$.

Remark 1.6. It turns out that $\ell_{\mu}=\left|\left\{i \leq k: d(\mu)_{i}>0\right\}\right|$-see Proposition 3.3.

1.7. Examples of initial cycles. Figure 2 illustrates ${ }^{\ddagger}$ two examples of 2 -graphs $\Lambda_{1}$ and $\Lambda_{2}$ containing lots of initial cycles. In fact, a cycle in either $\Lambda_{1}$ or $\Lambda_{2}$ is an initial cycle precisely if it contains edges of both colours. Each initial cycle in either $\Lambda_{i}$ visits every vertex, so any two initial cycles in $\Lambda_{i}$ are $\sim$-equivalent. A computation shows that for each initial cycle $\mu$ in either $\Lambda_{i}, G_{\mu} \cong \mathbb{Z}^{2}$. Notice that each vertex on a cycle in either $\Lambda_{i}$ has exactly one red (dashed) and one blue (solid) incoming and outgoing edge and exactly one infinite path with range at that vertex.

\footnotetext{
${ }^{\ddagger}$ We have illustrated $\Lambda_{1}$ and $\Lambda_{2}$ as 2-coloured graphs, we refer to [16] for details on how to visualise $k$-graphs as colours graphs.
} 
Below we illustrate how the stable rank of each $C^{*}\left(\Lambda_{i}\right)$ can be computed. For this we need some definitions and a lemma.

(1) Fix an integer $n \geq 1$, let $L_{n}$ denote the connected 1 -graph with $n$ vertices $v_{0}, \ldots, v_{n-1}$ and $n$ morphisms $f_{0}, \ldots, f_{n-1}$ of degree 1 such that $s\left(f_{i}\right)=$ $v_{i+1(\bmod n)}$ and $r\left(f_{i}\right)=v_{i}$ for $0 \leq i \leq n-1$.

(2) Let $\left(\Lambda_{1}, d_{1}\right)$ and $\left(\Lambda_{2}, d_{2}\right)$ be $k_{1^{-}}, k_{2}$-graphs respectively, then $\left(\Lambda_{1} \times \Lambda_{2}, d_{1} \times d_{2}\right)$ is a $\left(k_{1}+k_{2}\right)$-graph where $\Lambda_{1} \times \Lambda_{2}$ is the product category and $d_{1} \times d_{2}$ : $\Lambda_{1} \times \Lambda_{2} \rightarrow \mathbb{N}^{k_{1}+k_{2}}$ is given by $d_{1} \times d_{2}\left(\lambda_{1}, \lambda_{2}\right)=\left(d_{1}\left(\lambda_{1}\right), d_{2}\left(\lambda_{2}\right)\right) \in \mathbb{N}^{k_{1}} \times \mathbb{N}^{k_{2}}$ [21, Proposition 1.8].

(3) Let $f: \mathbb{N}^{\ell} \rightarrow \mathbb{N}^{k}$ be a monoid morphism. If $(\Lambda, d)$ is a $k$-graph we may form the $\ell$-graph $f^{*}(\Lambda)$ as follows: $f^{*}(\Lambda)=\{(\lambda, n): d(\lambda)=f(n)\}$ with $d(\lambda, n)=n, s(\lambda, n)=s(\lambda)$ and $r(\lambda, n)=r(\lambda)$ [21, Example 1.10].

(4) Let $\Lambda$ be a $k$-graph and define $g_{i}: \mathbb{N} \rightarrow \mathbb{N}^{k}$ by $g_{i}(n)=n e_{i}$ for $1 \leq i \leq k$ (so $\ell=1)$. The 1-graphs $\Lambda_{i}:=g_{i}^{*}(\Lambda)$ are called the coordinate graphs of $\Lambda$.

Lemma 1.7. [21, Proposition 1.11, Corollary 3.5(iii), Corollary 3.5(iv)]

(1) Let $\left(\Lambda_{i}, d_{i}\right)$ be $k_{i}$-graphs for $i=1,2$, then $C^{*}\left(\Lambda_{1} \times \Lambda_{2}\right) \cong C^{*}\left(\Lambda_{1}\right) \otimes C^{*}\left(\Lambda_{2}\right)$ via the map $s_{\left(\lambda_{1}, \lambda_{2}\right)} \mapsto s_{\lambda_{1}} \otimes s_{\lambda_{2}}$ for $\left(\lambda_{1}, \lambda_{2}\right) \in \Lambda_{1} \times \Lambda_{2}$.

(2) Let $\Lambda$ be a k-graph and $f: \mathbb{N}^{\ell} \rightarrow \mathbb{N}^{k}$ a surjective monoid morphism. Then $C^{*}\left(f^{*}(\Lambda)\right) \cong C^{*}(\Lambda) \otimes C\left(\mathbb{T}^{\ell-k}\right)$.

Let $\Lambda$ be a 1-graph and define $f_{1}: \mathbb{N}^{2} \rightarrow \mathbb{N}$ by $\left(m_{1}, m_{2}\right) \mapsto m_{1}+m_{2}$. Then $f_{1}^{*}\left(L_{6}\right)$ is isomorphic to the 2-graph $\Lambda$ shown on the left in Figure 2. The 2-graph $L_{6} \times L_{1}$ is isomorphic to the 2-graph shown on the right in Figure 2. Using Lemma 1.7 and that $C^{*}\left(L_{6}\right) \cong M_{6}(C(\mathbb{T}))\left[1\right.$, Lemma 2.4] we get $C^{*}\left(\Lambda_{i}\right) \cong C^{*}\left(L_{6}\right) \otimes C(\mathbb{T}) \cong M_{6}\left(C\left(\mathbb{T}^{2}\right)\right)$, $i=1,2$, so both have stable rank 2 as discussed in Section 1.1.

\section{Structure AND Stable RANK In the Stably Finite CASE.}

In this section we study finite $k$-graphs whose $C^{*}$-algebras are stably finite, corresponding to boxes 1 and 2 in Figure 1. In Proposition 2.7 we show that stable finiteness is equivalent to the lack of infinite projections and provide a characterisation in terms of properties of the $k$-graph. We also provide a structure result and compute stable rank of such $C^{*}$-algebras - see Theorems 2.5 and 2.6. We begin with four technical lemmas needed to prove Theorem 2.5.

Lemma 2.1. Let $\Lambda$ be a finite, locally convex $k$-graph that has no cycle with an entrance. Let $v \in \Lambda^{0}$ be a vertex on an initial cycle $\mu \in \Lambda$. Then

(1) there exist paths $\iota_{v}, \tau_{v} \in \Lambda$ such that $\mu=\iota_{v} \tau_{v}$ and $s\left(\iota_{v}\right)=v=r\left(\tau_{v}\right)$;

(2) the path $\mu_{v}:=\tau_{v} \iota_{v}$ satisfies $r\left(\mu_{v}\right)=s\left(\mu_{v}\right)$, and $r\left(\mu_{v}\right) \Lambda^{e_{i}}=\emptyset$ whenever $d\left(\mu_{v}\right)_{i}=0$

(3) if $f \in \Lambda^{e_{i}}$ is an edge with range $v$ on $\mu$, then $f=\mu_{v}\left(0, e_{i}\right)$ and $\mu=\nu^{\prime} f \nu^{\prime \prime}$ for some $\nu^{\prime}, \nu^{\prime \prime} \in \Lambda$;

(4) if $n \leq d(\mu)$ and $\lambda \in v \Lambda^{\leq n}$, then $\lambda=\mu_{v}(0, d(\lambda))$; and

(5) $s_{\tau_{v}} s_{\tau_{v}}^{*}=s_{v}$ and $s_{\tau_{v}}^{*} s_{\tau_{v}}=s_{s(\mu)}$.

Proof. (1). Since $v$ is a vertex on $\mu$, we have $v=\mu(p)$ for some $p \leq d(\mu)$. Set $\iota_{v}:=\mu(0, p)$ and $\tau_{v}:=\mu(p, d(\mu))$. Then $\mu=\iota_{v} \tau_{v}$ and $s\left(\iota_{v}\right)=v=r\left(\tau_{v}\right)$. 
(2). By property (1), $s\left(\iota_{v}\right)=v=r\left(\tau_{v}\right)$, so the path $\mu_{v}:=\tau_{v} \iota_{v} \in \Lambda$ satisfies $r\left(\mu_{v}\right)=s\left(\mu_{v}\right)$. Suppose $r\left(\mu_{v}\right) \Lambda^{e_{i}}$ is nonempty, say $\alpha \in r\left(\mu_{v}\right) \Lambda^{e_{i}}$. Then $\left(\iota_{v} \alpha\right)\left(0, e_{i}\right) \in$ $r(\mu) \Lambda^{e_{i}}$. Since $\mu$ is an initial cycle, it follows that $d(\mu)_{i} \neq 0$.

(3). Suppose $f \in \Lambda^{e_{i}}$ is an edge with range $v$ on $\mu$. Since $r(f)=v=r\left(\mu_{v}\right)$, we have $f \in r\left(\mu_{v}\right) \Lambda^{e_{i}}$. Now property (2) ensures that $d\left(\mu_{v}\right)_{i} \neq 0$. Since $d\left(\mu_{v}\right)_{i}>0$, there exists a path $\lambda \in s(f) \Lambda \leq d\left(\mu_{v}\right)-e_{i}$. Hence $f \lambda \in \Lambda^{\leq e_{i}} \Lambda^{\leq d\left(\mu_{v}\right)-e_{i}}=\Lambda^{\leq d\left(\mu_{v}\right)}$. Now using that $\mu_{v} \in v \Lambda$ is a cycle and that $\Lambda$ has no cycle with an entrance, it follows that $v \Lambda \leq d\left(\mu_{v}\right)=\left\{\mu_{v}\right\}$. Hence $\mu_{v}=f \lambda$. Since $f \lambda=\mu_{v}=\tau_{v} \iota_{v}$, we get $f=\tau_{v}\left(0, e_{i}\right)$ if $d\left(\tau_{v}\right)_{i}>0$ and $f=\iota_{v}\left(0, e_{i}\right)$ if $d\left(\tau_{v}\right)_{i}=0$.

(4). Fix $n \leq d(\mu)$ and $\lambda \in v \Lambda^{\leq n}$. If $d(\lambda)=0$ then $\lambda=v$ and the statement is trivial, so we may assume that $\lambda \notin \Lambda^{0}$. Write $d(\lambda)=e_{i_{1}}+\cdots+e_{i_{m}}$ where $i_{1} \ldots, i_{m} \in\{1, \ldots, k\}$. By the factorisation property $\lambda=\lambda_{1} \ldots \lambda_{m}$ for some $\lambda_{j} \in$ $\Lambda^{e_{i_{j}}}$. Repeated applications of part (3) give $\lambda_{j}=\mu_{r\left(\lambda_{j}\right)}\left(0, e_{i_{j}}\right)$ for $j \leq m$. Since $d(\lambda) \leq d(\mu)$, it follows that $\lambda=\mu_{v}(0, d(\lambda))$.

(5). Since $s\left(\tau_{v}\right)=s(\mu)$ we have $s_{\tau_{v}}^{*} s_{\tau_{v}}=s_{s(\mu)}$. For $n:=d\left(\tau_{v}\right)$ notice that $n \leq d(\mu)$. Fix $\lambda \in v \Lambda^{\leq n}$. Using part (4) we have $\lambda=\mu_{v}(0, d(\lambda))$. Since $\tau_{v} \in v \Lambda^{\leq n}$ and since $d(\lambda) \leq n$ we have $\tau_{v}=\mu_{v}(0, n)=\lambda \mu_{v}(d(\lambda), n)$. But both $\tau_{v}$ and $\lambda$ belong to $\Lambda \leq n$, so $\tau_{v}=\lambda$. Consequently $v \Lambda^{\leq n}=\left\{\tau_{v}\right\}$ and $s_{\tau_{v}} s_{\tau_{v}}^{*}=\sum_{\lambda \in v \Lambda \leq n} s_{\lambda} s_{\lambda}^{*}=s_{v}$.

Lemma 2.2. Let $\Lambda$ be a finite, locally convex $k$-graph that has no cycle with an entrance. Let $N:=\left(\left|\Lambda^{0}\right|, \ldots,\left|\Lambda^{0}\right|\right) \in \mathbb{N}^{k}$. Then

(1) $\sum_{\lambda \in \Lambda \leq N} s_{\lambda} s_{\lambda}^{*}=1_{C^{*}(\Lambda)}$; and

(2) for every $\lambda \in \Lambda^{\leq N}, s(\lambda)$ is a vertex on an initial cycle.

Proof. For part (1) use $1=\sum_{v \in \Lambda^{0}} s_{v}=\sum_{v \in \Lambda^{0}} \sum_{\lambda \in v \Lambda \leq N} s_{\lambda} s_{\lambda}^{*}=\sum_{\lambda \in \Lambda \leq N} s_{\lambda} s_{\lambda}^{*}$. For part (2) we refer to the second paragraph of the proof of [14, Proposition 5.9].

Recall that $\left(\mu^{\infty}\right)^{0}$ denotes the collection of all vertices on an initial cycle $\mu$. For the terminology $\tau_{v}, v \in \Lambda^{0}$ in the following lemma see Lemma 2.1.

Lemma 2.3. Let $\Lambda$ be a finite, locally convex $k$-graph that has no cycle with an entrance. Fix an initial cycle $\mu \in \Lambda$. Let $N:=\left(\left|\Lambda^{0}\right|, \ldots,\left|\Lambda^{0}\right|\right) \in \mathbb{N}^{k}$ and for each $\lambda, \nu \in \Lambda^{\leq N}\left(\mu^{\infty}\right)^{0}$ set (using Lemma 2.1)

$$
\theta_{\lambda, \nu}:=s_{\lambda \tau_{s(\lambda)}} s_{\nu \tau_{s(\nu)}}^{*}
$$

Then the $\theta_{\lambda, \nu}$ are matrix units, i.e., $\theta_{\lambda, \nu}^{*}=\theta_{\nu, \lambda}$ and $\theta_{\lambda, \nu} \theta_{\gamma, \eta}=\delta_{\nu, \gamma} \theta_{\lambda, \eta}$ in $C^{*}(\Lambda)$.

Proof. Firstly, note that each $\theta_{\lambda, \nu}$ makes sense because the source of $\lambda \in \Lambda^{\leq N}\left(\mu^{\infty}\right)^{0}$ is a vertex on $\mu$ and $\tau_{s(\lambda)}$ is a path on the initial cycle $\mu$ with range $s(\lambda)$.

Fix $\lambda, \nu, \gamma, \eta \in \Lambda^{\leq N}\left(\mu^{\infty}\right)^{0}$. Clearly $\theta_{\lambda, \nu}^{*}=\theta_{\nu, \lambda}$. We claim that $\theta_{\lambda, \nu} \theta_{\gamma, \eta}=\delta_{\nu, \gamma} \theta_{\lambda, \eta}$. To see this let $v:=r(\mu)$. Then

$$
s_{\nu \tau_{s(\nu)}}^{*} s_{\gamma \tau_{s(\gamma)}}=s_{\tau_{s(\nu)}}^{*} s_{\nu}^{*} s_{\gamma} s_{\tau_{s(\gamma)}}
$$

is nonzero only if $r(\nu)=r(\gamma)$. Since $s_{r(\nu)}=\sum_{\alpha \in r(\nu) \Lambda \leq N} s_{\alpha} s_{\alpha}^{*}$, we have $\left(s_{\nu} s_{\nu}^{*}\right)\left(s_{\gamma} s_{\gamma}^{*}\right)=$ $\delta_{\nu, \gamma} s_{\nu} s_{\nu}^{*}$, so if (2.1) is nonzero, then $\nu=\gamma$ and then

$$
s_{\nu \tau_{s(\nu)}}^{*} s_{\gamma \tau_{s(\gamma)}}=\delta_{\nu, \gamma} s_{\nu \tau_{s(\nu)}}^{*} s_{\gamma \tau_{s(\gamma)}}=\delta_{\nu, \gamma} s_{s\left(\nu \tau_{s(\nu)}\right)}=\delta_{\nu, \gamma} s_{s\left(\tau_{s(\nu)}\right)}=\delta_{\nu, \gamma} s_{v} .
$$

Hence $\theta_{\lambda, \nu} \theta_{\gamma, \eta}=s_{\lambda \tau_{s(\lambda)}}\left(\delta_{\nu, \gamma} s_{v}\right) s_{\eta \tau_{s(\eta)}}^{*}=\delta_{\nu, \gamma} \theta_{\lambda, \eta}$ as claimed. 
The next lemma is of general nature. See [22, Lemma 3.3] for a special case of this result.

Lemma 2.4. Suppose that $\left\{e_{i j}^{(k)}: 1 \leq k \leq r, 1 \leq i, j \leq n_{k}\right\}$ is a system of matrix units in a unital $C^{*}$-algebra $A$, in the sense that

(1) $e_{i j}^{(k)} e_{j l}^{(k)}=e_{i l}^{(k)}$;

(2) $e_{i j}^{(k)} e_{m n}^{(l)}=0$ if $k \neq l$ or if $j \neq m$;

(3) $\left(e_{i j}^{(k)}\right)^{*}=e_{j i}^{(k)}$; and

(4) $\sum_{k=1}^{r} \sum_{i=1}^{n_{k}} e_{i i}^{(k)}=1$.

For $k \leq r$ let $p^{(k)}:=\sum_{i=1}^{n_{k}} e_{i i}^{(k)}$. Suppose that for each $a \in A, a=\sum_{k=1}^{r} p^{(k)} a p^{(k)}$. Then, for $1 \leq k \leq r$, each $e_{11}^{(k)}$ is a projection and $A \cong \bigoplus_{k=1}^{r} M_{n_{k}}\left(e_{11}^{(k)} A e_{11}^{(k)}\right)$.

Proof. Clearly $A \cong \bigoplus_{k=1}^{r} p^{(k)} A p^{(k)}$ via $a \mapsto\left(p^{(1)} a p^{(1)}, \ldots, p^{(r)} a p^{(r)}\right)$ and inverse $\left(a^{(1)}, \ldots, a^{(r)}\right) \mapsto \sum_{k=1}^{r} a^{(k)}$. Routine calculations show that for each $k \in\{1, \ldots, r\}$, the elements $v_{i}:=e_{i 1}^{(k)}, i=1, \ldots, n_{k}$, satisfy

$$
v_{i}^{*} v_{j}=\delta_{i, j} e_{11}^{(k)} \quad \text { for } \quad 1 \leq i, j \leq n_{k}, \quad \text { and } \quad p^{(k)}=\sum_{i=1}^{n_{k}} v_{i} v_{i}^{*} .
$$

By [22, Lemma 3.3], $p^{(k)} A p^{(k)} \cong M_{n_{k}}\left(e_{11}^{(k)} A e_{11}^{(k)}\right)$ completing the proof.

We now characterise the structure of $k$-graph $C^{*}$-algebras $C^{*}(\Lambda)$ such that $\Lambda$ is finite and has no cycle with an entrance. For the notions of $\operatorname{IC}(\Lambda), \sim,\left(\mu^{\infty}\right)^{0}$, and $\ell_{\mu}$ see Section 1.6. We note that in Theorem 2.5, $\operatorname{IC}(\Lambda) \neq \emptyset$ and $\ell_{\mu}=\mid\left\{i \leq k: d(\mu)_{i}>\right.$ $0\} \mid$ (see Lemma 2.2(2) and Proposition 3.3).

Theorem 2.5. (Structure theorem) Let $\Lambda$ be a finite, locally convex $k$-graph that has no cycle with an entrance. For $N:=\left(\left|\Lambda^{0}\right|, \ldots,\left|\Lambda^{0}\right|\right) \in \mathbb{N}^{k}$,

$$
C^{*}(\Lambda) \cong \bigoplus_{[\mu] \in \operatorname{IC}(\Lambda) / \sim} M_{\Lambda \leq N\left(\mu^{\infty}\right)^{0}}\left(s_{r(\mu)} C^{*}(\Lambda) s_{r(\mu)}\right)
$$

and each $s_{r(\mu)} C^{*}(\Lambda) s_{r(\mu)} \cong C\left(\mathbb{T}^{\ell_{\mu}}\right)$.

Proof. Evidently $\operatorname{IC}(\Lambda) / \sim$ is finite since $\Lambda^{0}$ is finite. Let $I$ be a maximal collection of initial cycles satisfying $\left(\mu^{\infty}\right)^{0} \cap\left(\nu^{\infty}\right)^{0}=\emptyset$ for any $\mu \neq \nu \in I$. For each initial cycle $\mu \in I$ let $\left\{\theta_{\lambda, \nu}^{(\mu)}\right\}$ be the matrix units of Lemma 2.3. We first prove that $\left\{\theta_{\lambda, \nu}^{(\mu)}: \mu \in I\right\}$ is a system of matrix units, i.e.,

(1) $\theta_{\lambda \lambda^{\prime}}^{(\mu)} \theta_{\lambda^{\prime} \lambda^{\prime \prime}}^{(\mu)}=\theta_{\lambda \lambda^{\prime \prime}}^{(\mu)}$

(2) $\theta_{\lambda \lambda^{\prime}}^{(\mu)} \theta_{\eta \eta^{\prime}}^{(\nu)}=0$ if $\mu \neq \nu$ or if $\lambda^{\prime} \neq \eta$;

(3) $\left(\theta_{\lambda \lambda^{\prime}}^{(\mu)}\right)^{*}=\theta_{\lambda^{\prime} \lambda}^{(\mu)}$; and

(4) $\sum_{\mu \in I} \sum_{\lambda \in \Lambda \leq N\left(\mu^{\infty}\right)^{0}} \theta_{\lambda \lambda}^{(\mu)}=1$.

We start with property (4). Fix $\mu \in I$ and $\lambda \in \Lambda^{\leq N}\left(\mu^{\infty}\right)^{0}$. Using Lemma 2.1(5) we have $s_{\tau_{s(\lambda)}} s_{\tau_{s(\lambda)}}^{*}=s_{s(\lambda)}$. Hence $\theta_{\lambda, \lambda}^{(\mu)}=s_{\lambda \tau_{s(\lambda)}} s_{\lambda \tau_{s(\lambda)}}^{*}=s_{\lambda} s_{\lambda}^{*}$. Lemma 2.2(1) now gives

$$
1=\sum_{\lambda \in \Lambda \leq N} s_{\lambda} s_{\lambda}^{*}=\sum_{\mu \in I} \sum_{\lambda \in \Lambda \leq N\left(\mu^{\infty}\right)^{0}} s_{\lambda} s_{\lambda}^{*}=\sum_{\mu \in I} \sum_{\lambda \in \Lambda \leq N\left(\mu^{\infty}\right)^{0}} \theta_{\lambda \lambda}^{(\mu)} .
$$


Properties (1)-(3) follow from Lemma 2.2 and that $\theta_{\lambda \lambda}^{(\mu)} \theta_{\lambda \lambda}^{(\nu)}=0$ whenever $\mu \neq \nu$ in $I$ (the latter is a consequence of property (4)).

For each $\mu \in I$, define $p^{(\mu)}:=\sum_{\lambda \in \Lambda \leq N\left(\mu^{\infty}\right)^{0}} \theta_{\lambda \lambda}^{(\mu)}$. Then (2.2) gives $\sum_{\mu \in I} p^{(\mu)}=1$. We claim that

$$
A:=\left\{a \in C^{*}(\Lambda): a=\sum_{\mu \in I} p^{(\mu)} a p^{(\mu)}\right\}
$$

is all of $C^{*}(\Lambda)$. Clearly $A$ is a closed linear subspace of $C^{*}(\Lambda)$. Fix $\alpha, \beta \in \Lambda^{\leq N}$ such that $s(\alpha)=s(\beta)$. Using Lemma 2.2(2) it follows that $s(\alpha) \in\left(\mu^{\infty}\right)^{0}$ for some $\mu \in I$. Since $s_{\alpha} s_{\alpha}^{*} \leq p^{(\mu)}$ we get

$$
s_{\alpha}=p^{(\mu)} s_{\alpha} s_{\alpha}^{*} s_{\alpha}=p^{(\mu)} s_{\alpha}, \quad \text { and } s_{\alpha} s_{\beta}^{*}=p^{(\mu)} s_{\alpha} s_{\beta}^{*} p^{(\mu)},
$$

so $s_{\alpha} s_{\beta}^{*} \in A$. Using that $\operatorname{span}\left\{s_{\alpha} s_{\beta}^{*}: \alpha, \beta \in \Lambda^{\leq N}, s(\alpha)=s(\beta)\right\}$ is dense in $C^{*}(\Lambda)$ we get $A=C^{*}(\Lambda)$ as claimed.

For each $\mu \in I$, Lemma 2.1(3)-(4) implies that $r(\mu) \Lambda^{\leq N}$ contains exactly one path which we denote by $\lambda_{\mu}$. As in the proof of (4), we have $\theta_{\lambda_{\mu}, \lambda_{\mu}}^{(\mu)}=s_{\lambda_{\mu}} s_{\lambda_{\mu}}^{*}$, so $s_{r(\mu)}=\sum_{\lambda \in r(\mu) \Lambda \leq N} s_{\lambda} s_{\lambda}^{*}=\theta_{\lambda_{\mu}, \lambda_{\mu}}^{(\mu)}$. Identifying $I$ with $\operatorname{IC}(\Lambda) / \sim$ via the map $\mu \mapsto[\mu]$, Lemma 2.4 provides an isomorphism

$$
C^{*}(\Lambda) \cong \bigoplus_{\mu \in \operatorname{IC}(\Lambda) / \sim} M_{\Lambda \leq N\left(\mu^{\infty}\right)^{0}}\left(s_{r(\mu)} C^{*}(\Lambda) s_{r(\mu)}\right) .
$$

To see that each $s_{r(\mu)} C^{*}(\Lambda) s_{r(\mu)} \cong C\left(\mathbb{T}^{\ell \mu}\right)$, see the proof of [14, Proposition 5.9].

The main result of this section is the characterisation of stable rank for $k$-graph $C^{*}$-algebras $C^{*}(\Lambda)$ such that $\Lambda$ is finite and has no cycle with an entrance. Recall the notion of the floor and ceiling functions: for $x \in \mathbb{R}$, we write $\lfloor x\rfloor:=\max \{n \in$ $\mathbb{Z}: n \leq x\}$ and $\lceil x\rceil:=\min \{n \in \mathbb{Z}: n \geq x\}$.

Theorem 2.6. Let $\Lambda$ be a finite, locally convex $k$-graph that has no cycle with an entrance. For $N:=\left(\left|\Lambda^{0}\right|, \ldots,\left|\Lambda^{0}\right|\right) \in \mathbb{N}^{k}$,

$$
\operatorname{sr}\left(C^{*}(\Lambda)\right)=\max _{[\mu] \in \operatorname{IC}(\Lambda) / \sim}\left\lceil\frac{\left\lfloor\frac{\ell_{\mu}}{2}\right\rfloor}{\left|\Lambda \leq N\left(\mu^{\infty}\right)^{0}\right|}\right\rceil+1 .
$$

Proof. By Theorem 2.5 and property (4) from Section 1.1,

$$
s r\left(C^{*}(\Lambda)\right)=\max _{[\mu] \in \operatorname{IC}(\Lambda) / \sim} M_{\Lambda \leq N\left(\mu^{\infty}\right)^{0}}\left(s_{r(\mu)} C^{*}(\Lambda) s_{r(\mu)}\right),
$$

and each $s_{r(\mu)} C^{*}(\Lambda) s_{r(\mu)} \cong C\left(\mathbb{T}^{\ell_{\mu}}\right)$. Now using property (2) from Section 1.1, we get

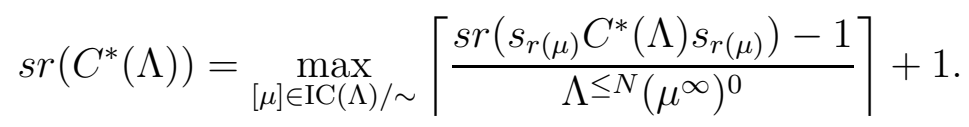

Finally, property (1) from Section 1.1, gives $\operatorname{sr}\left(s_{r(\mu)} C^{*}(\Lambda) s_{r(\mu)}\right)-1=\left\lfloor\ell_{\mu} / 2\right\rfloor$ for each $[\mu] \in \operatorname{IC}(\Lambda) / \sim$, completing the proof.

Our next Proposition 2.7 characterises stable finiteness of $C^{*}$-algebras of finite, locally convex $k$-graphs. For other results on stable finiteness of $C^{*}$-algebras associated to row-finite $k$-graphs with no sources, see $[7,14]$. Note that the $C^{*}$-algebras satisfying the hypotheses of Proposition 2.7 are exactly those shown in Figure 1 in boxes 3 and 4 . 
We briefly introduce relevant terminology. Following [31] we write $\operatorname{MCE}(\mu, \nu):=$ $\mu \Lambda \cap \nu \Lambda \cap \Lambda^{d(\mu) \vee d(\nu)}$ for the set of all minimal common extensions of $\mu, \nu \in \Lambda$. The cycle $\lambda$ is a cycle with an entrance in the sense of [14, Definition 3.5] if there exists a path $\tau \in r(\lambda) \Lambda$ such that $\operatorname{MCE}(\tau, \lambda)=\emptyset \S$.

Proposition 2.7. Let $\Lambda$ be a finite, locally convex $k$-graph. With notation as above, the following are equivalent:

(1) $\Lambda$ has a cycle $\mu$ with an entrance;

(2) $\Lambda$ has a cycle $\mu$ with an entrance in the sense of [14, Definition 3.5];

(3) $C^{*}(\Lambda)$ contains an infinite projection; and

(4) $C^{*}(\Lambda)$ is not stably finite.

Proof. To prove $(1) \Rightarrow(2)$ let $\mu$ be a cycle with an entrance $\tau$, so $\tau \in r(\mu) \Lambda$ satisfies $d(\tau) \leq d\left(\mu^{\infty}\right)$ and $\tau \neq \mu^{\infty}(0, d(\tau))$. Fix $n \geq 1$ such that $n d(\mu) \geq d(\tau)$. Clearly $\tau \in$ $r\left(\mu^{n}\right) \Lambda$. Then $\tau \neq \mu^{\infty}(0, d(\tau))=\mu^{n}(0, d(\tau))$, so $\operatorname{MCE}\left(\mu^{n}, \tau\right)=\left(\mu^{n} \Lambda \cap \Lambda^{d\left(\mu^{n}\right) \vee d(\tau)}\right) \cap$ $\tau \Lambda \subseteq\left\{\mu^{n}\right\} \cap \tau \Lambda=\emptyset$. For the proof of $(2) \Rightarrow(3)$, see [14, Corollary 3.8].

The implication $(3) \Rightarrow(4)$ follows from [37, Lemma 5.1.2]. It remains to prove $(4) \Rightarrow(1)$. We establish the contrapositive. Suppose that condition (1) does not hold, that is $\Lambda$ has no cycle with an entrance. Theorem 2.5 gives that $C^{*}(\Lambda)$ is isomorphic to a direct sum of matrix algebras over commutative $C^{*}$-algebras, hence stably finite, so condition (4) does not hold.

Remark 2.8. Our main results are for finite $k$-graphs so Proposition 2.7 is stated in that context, but some of the implications hold more generally.

(1) Only the proof of (4) $\Longrightarrow(1)$ uses that $\Lambda$ is finite. The proofs of the implications $(1) \Longrightarrow(2) \Longrightarrow(3) \Longrightarrow(4)$ are valid for any locally convex row-finite $k$-graph.

(2) Similarly, while (1) and (2) are not equivalent for arbitrary $k$-graphs, they are equivalent for locally convex $k$-graphs, whether finite or not. To see this, suppose that $\lambda$ is a cycle in a locally convex $k$-graph and $\tau \in r(\lambda) \Lambda$ satisfies $\operatorname{MCE}(\lambda, \tau)=\emptyset$. Let $I=\left\{i \leq k: d(\lambda)_{i}>0\right\}$, let $m_{I}=\sum_{i \in I} d(\tau)_{i} e_{i}$ and $m^{\prime}:=d(\tau)-m_{I}$, and factorise $\tau=\tau_{I} \tau^{\prime}$ with $d\left(\tau_{I}\right)=m_{I}$. If $\tau_{I} \neq\left(\lambda^{\infty}\right)\left(0, m_{I}\right)$, then $\lambda$ is a cycle with an entrance as required, so we may assume that $\tau_{I}=\left(\lambda^{\infty}\right)\left(0, m_{I}\right)$. So replacing $\lambda$ with $\left(\lambda^{\infty}\right)\left(m_{I}, m_{I}+d(\lambda)\right)$ and $\tau$ with $\tau^{\prime}$ we may assume that $d(\tau) \wedge d(\lambda)=0$. Since $\Lambda$ is locally convex, a quick inductive argument shows that there exists $\mu \in s(\tau) \Lambda^{d(\lambda)} \neq \emptyset$. Factorise $\tau \mu=\alpha \beta$ with $d(\alpha)=d(\mu)=d(\lambda)$. Since $\operatorname{MCE}(\tau, \lambda)=\emptyset$, we must have $\alpha \neq \lambda$ and in particular $d(\alpha)=d(\lambda)<d\left(\lambda^{\infty}\right)$ and $\alpha \neq\left(\lambda^{\infty}\right)(0, d(\alpha))$. So once again $\lambda$ is a cycle with an entrance.

Corollary 2.9. Let $\Lambda$ be a finite, locally convex $k$-graph. Suppose that $\Lambda$ has no cycle with an entrance (i.e., $C^{*}(\Lambda)$ is stably finite). For $N:=\left(\left|\Lambda^{0}\right|, \ldots,\left|\Lambda^{0}\right|\right) \in \mathbb{N}^{k}$,

$$
\operatorname{sr}\left(C^{*}(\Lambda)\right)=\max _{[\mu] \in \operatorname{IC}(\Lambda) / \sim}\left\lceil\frac{\left\lfloor\frac{\ell_{\mu}}{2}\right\rfloor}{\left|\Lambda \leq N\left(\mu^{\infty}\right)^{0}\right|}\right\rceil+1 .
$$

\footnotetext{
§Formally, if $\lambda$ is a cycle, then $(\lambda, r(\lambda))$ is a generalised cycle in the sense of [14, Definition 3.1], and an entrance to $(\lambda, r(\lambda))$ is a path $\tau \in s(r(\lambda)) \Lambda$ such that $\operatorname{MCE}(r(\lambda) \tau, \lambda)=\emptyset$.
} 


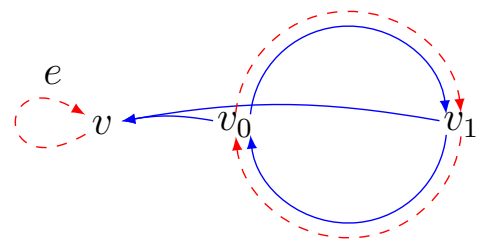

Figure 3. An example of a 2-graph $\Lambda$ with $C^{*}(\Lambda)$ of stable rank 2.

Remark 2.10. A cycle with an incoming edge may fail to be a cycle with an entrance. This is for example the case for any of the red (dashed) cycles in Figure 3.

Example 2.11. In this example we consider the 2-graph $\Lambda$ in Figure 3. As before, we refer to [16] for details on how to illustrate 2-graphs as a 2-coloured graph. Here we use blue (solid) and red (dashed) as the first and second colour. We use our results to compute the structure and stable rank of $C^{*}(\Lambda)$. Firstly notice that red the cycle $\nu \in v \Lambda^{e_{2}}$ based on $v$ is not an initial cycle because, $d(\nu)_{1}=0$, but $r(\nu) \Lambda^{e_{1}} \neq \emptyset$. However, the cycle $\mu \in v_{0} \Lambda^{e_{1}+e_{2}}$ is an initial cycle. There are many other initial cycles, but they are all $\sim$-equivalent to $\mu$. So $\operatorname{IC}(\Lambda) / \sim=\{[\mu]\}$. Since the vertices on a path $\lambda \in v_{0} \Lambda$ alternate between $v_{0}$ and $v_{1}$ as we move along the path, it follows that $\mu^{\infty}(m)=v_{m_{1}+m_{2}}(\bmod 2)$ for each $m \in \mathbb{N}^{2}$. Hence

$$
\begin{aligned}
G_{\mu} & =\left\{m-n: n, m \leq d\left(\mu^{\infty}\right), \mu^{\infty}(m)=\mu^{\infty}(n)\right\} \\
& =\left\{m-n: n, m \in \mathbb{N}^{2}, v_{m_{1}+m_{2}} \quad(\bmod 2)=v_{n_{1}+n_{2}} \quad(\bmod 2)\right\} \\
& =\left\{\left(m_{1}-n_{1}, m_{2}-n_{2}\right): n_{i}, m_{i} \in \mathbb{N}, m_{1}-n_{1}=-\left(m_{2}-n_{2}\right) \quad(\bmod 2)\right\} \\
& =\left\{\left(k_{1}, k_{2}\right): k_{i} \in \mathbb{Z}, k_{1}=-k_{2} \quad(\bmod 2)\right\} \\
& =\left\{k \in \mathbb{Z}^{2}: k_{1}+k_{2} \text { is even }\right\} \\
& =\mathbb{Z}(1,1)+\mathbb{Z}(0,2), \\
& \cong \mathbb{Z}^{2} .
\end{aligned}
$$

We deduce that $\ell_{\mu}=\operatorname{rank}\left(G_{\mu}\right)=2$. Now set $N=\left(\left|\Lambda^{0}\right|,\left|\Lambda^{0}\right|\right)=(3,3)$. As mentioned, modulo $\sim$, there is only one initial cycle $\mu$, so any path in $\Lambda^{\leq N}$ has its source on $\mu$. Hence $\Lambda^{\leq N}\left(\mu^{\infty}\right)^{0}=\Lambda^{\leq N}=v \Lambda^{\leq N} \sqcup v_{0} \Lambda^{\leq N} \sqcup v_{1} \Lambda^{\leq N}$. By Lemma 2.1, $\left|v_{0} \Lambda^{\leq N}\right|=$ $\left|v_{1} \Lambda^{\leq N}\right|=1$. Using the factorisation property to push the red edges to the start of a path and uniqueness of such paths on $\mu$, we have $\left|v \Lambda^{\leq N}\right|=\left|v \Lambda^{(3,3)}\right|=\left|v \Lambda^{(3,0)}\right|=$ $\left|v \Lambda^{(1,0)}\right|=2$. Hence $C^{*}(\Lambda) \cong M_{4}\left(C\left(\mathbb{T}^{2}\right)\right)$ and $\operatorname{sr}\left(C^{*}(\Lambda)\right)=\left\lceil\left\lfloor\frac{2}{2}\right\rfloor / 4\right\rceil+1=2$ by Theorem 2.6.

Example 2.12. In the following let $\Lambda_{1}, \Lambda_{2}$ and $\Lambda_{3}, \Lambda_{4}, \Lambda_{5}$ be the 2-graphs in Figure 2 and Figure 4 respectively. Up to a swap of the colours these five examples make up all the examples of 2 -graphs on 6 vertices with only one initial cycle up to $\sim$ equivalence and with all vertices on that initial cycle. Let $\mu_{i}$ denote such an initial cycle in $\Lambda_{i}$. For $0 \leq j \leq n-1$ define $f_{j}: \mathbb{N}^{2} \rightarrow \mathbb{N}$ by $f_{j}\left(m_{1}, m_{2}\right)=m_{1}+j m_{2}$. With the terminology of Section 1.7 one can show that $\Lambda_{3}=f_{5}^{*}\left(L_{6}\right)$, that $\Lambda_{4}=L_{2} \times L_{3}$, and that $\Lambda_{5}=f_{2}^{*}\left(L_{6}\right)$. Hence

$$
C^{*}\left(\Lambda_{i}\right) \cong M_{6}\left(C\left(\mathbb{T}^{\ell_{\mu_{i}}}\right)\right) \text { and } \operatorname{sr}\left(C^{*}(\Lambda)\right)=\ell_{\mu_{i}}=2 \quad \text { for each } i=1, \ldots, 5 .
$$



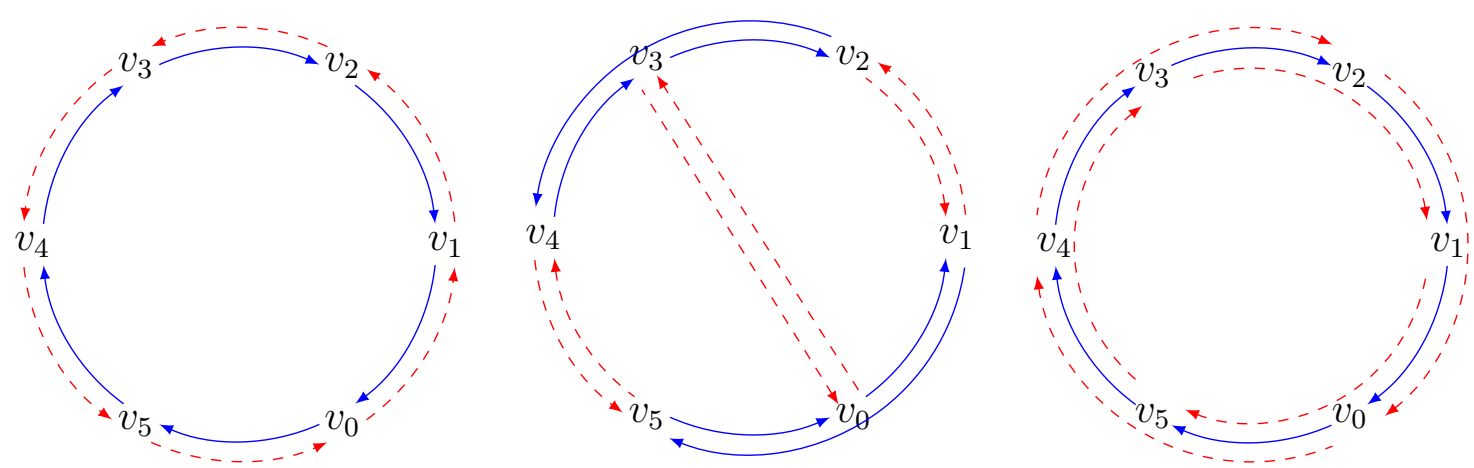

FiguRE 4. Another three examples of 2-graphs with lots of initial cycles, but only one such up to $\sim$-equivalence.

In particular $C^{*}\left(\Lambda_{i}\right) \cong C^{*}\left(\Lambda_{j}\right)$ for all $i, j$. These five examples indicate how the number of colours and vertices impacts the structure of the corresponding $C^{*}$-algebras. The next Proposition 2.13 verifies this.

In the following, "the number of vertices" on an initial cycle $\mu$ means $\left|\left(\mu^{\infty}\right)^{0}\right|$, and "the number of colours" means $\left|\left\{i \leq k: d(\mu)_{i}>0\right\}\right|$. The proof borrows material from an independent result (Proposition 3.3).

Proposition 2.13. Let $\Lambda$ be a finite, locally convex $k$-graph on $n=\left|\Lambda^{0}\right|$ vertices. Suppose that $\Lambda$ has no cycle with an entrance and $\Lambda$ has exactly one initial cycle, up to $\sim$-equivalence, with $n$ vertices and $\ell$ colours. Then

$$
C^{*}(\Lambda) \cong M_{n}\left(C\left(\mathbb{T}^{\ell}\right)\right) \text {, and } \operatorname{sr}\left(C^{*}(\Lambda)\right)=\lceil\lfloor\ell / 2\rfloor / n\rceil+1 .
$$

Proof. Let $\mu$ be an initial cycle in $\Lambda$. By Theorem 2.5 and Theorem 2.6 we have

$$
s r\left(C^{*}(\Lambda)\right)=\left\lceil\frac{\left\lfloor\frac{\ell_{\mu}}{2}\right\rfloor}{|\Lambda \leq(n, \ldots, n)|}\right\rceil+1, \quad C^{*}(\Lambda) \cong M_{\Lambda \leq(n, \ldots, n)}\left(C\left(\mathbb{T}^{\ell_{\mu}}\right)\right),
$$

where $\ell_{\mu}$ is the rank of the periodicity group associated to $\mu$ (Definition 1.5). By the factorisation property $\Lambda$ has no sources, so $\Lambda^{\leq(n, \ldots, n)}=\Lambda^{(n, \ldots, n)}$. Lemma 2.1(3) implies that $\left|\Lambda^{(n, \ldots, n)}\right|=n$. By Proposition 3.3, $\ell_{\mu}=\ell$. Combining these results gives $C^{*}(\Lambda) \cong M_{n}\left(C\left(\mathbb{T}^{\ell}\right)\right)$, and $\operatorname{sr}\left(C^{*}(\Lambda)\right)=\lceil\lfloor\ell / 2\rfloor / n\rceil+1$.

Remark 2.14. To keep the statement of Proposition 2.13 short and clean we insisted that $\left(\mu^{\infty}\right)^{0}=\Lambda^{0}$, but more general results can be obtained using Theorem 2.5 and Theorem 2.6.

\section{STABLE RANK ONE.}

In this section we characterise which finite $k$-graphs have $C^{*}$-algebras of stable rank 1 - see Theorem 3.1 and Corollary 3.4. We note that Theorem 3.1 is in large contained in [14] and we have structured the proof accordingly.

Theorem 3.1. Let $\Lambda$ be a finite, locally convex $k$-graph. Then $\operatorname{sr}\left(C^{*}(\Lambda)\right)=1$ if and only if $C^{*}(\Lambda)$ is (stably) finite and $\max _{\mu \in I C(\Lambda)} \ell_{\mu}=1$. 


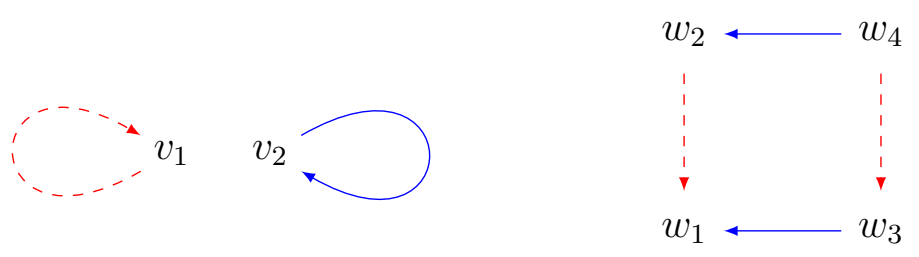

Figure 5. Two examples of 2-graphs with a $C^{*}$-algebra of stable rank one.

Proof. Suppose that $\operatorname{sr}\left(C^{*}(\Lambda)\right)=1$. Then $\operatorname{sr}\left(M_{n}\left(C^{*}(\Lambda)\right)\right)=1$ for each positive integer $n$ [34, Theorem 6.1]. Hence each $M_{n}\left(C^{*}(\Lambda)\right)$ is finite [2, V.3.1.5], which implies that $C^{*}(\Lambda)$ is stably finite. Since $C^{*}(\Lambda)$ is finite it does not contain any infinite projections [37, Lemma 5.1.2]. Hence by [14, Proposition 5.9] there exist $n \geq$ 1 and $l_{1}, \ldots, l_{n} \in\{0, \ldots, k\}$ such that $C^{*}(\Lambda)$ is stably isomorphic to $\bigoplus_{i=1}^{n} C\left(\mathbb{T}^{l_{i}}\right)$. Since $\operatorname{sr}\left(C^{*}(\Lambda)\right)=1$, we deduce that $\operatorname{sr}\left(\bigoplus_{i=1}^{n} C\left(\mathbb{T}^{l}\right)\right)=1$, because stable rank 1 for unital $C^{*}$-algebras is preserved my stable isomorphism [34, Theorem 3.6]. By property 4 in Section 1.1, we have $\operatorname{sr}\left(\bigoplus_{i=1}^{n} C\left(\mathbb{T}^{l_{i}}\right)\right)=\max _{i=1}^{n} C\left(\mathbb{T}^{l_{i}}\right)$. For each $i=1, \ldots, n$ we use $\left[34\right.$, Proposition 1.7] to deduce that $\operatorname{sr}\left(C\left(\mathbb{T}^{l_{i}}\right)\right)=\left\lfloor l_{i} / 2\right\rfloor+1$, where $\lfloor\cdot\rfloor$ denotes "integer part of". Hence $\max _{i=1}^{n} l_{i}=1$.

By inspection of the proof of [14, Proposition 5.9] it is clear that each of the integers $l_{i}$ is the rank of $\mu$ for some $\mu \in I C(\Lambda)$, so $\max _{\mu \in I C(\Lambda)} \ell_{\mu} \geq \max _{i=1}^{n} l_{i}=1$. For each $\mu, \nu \in I C(\Lambda)$ define $P_{\nu}:=\sum_{v \in\left(\nu^{\infty}\right)^{0}} s_{v}$ and $\mu \sim \nu \Leftrightarrow\left(\mu^{\infty}\right)^{0}=\left(\nu^{\infty}\right)^{0}$. Since $P_{\nu}=P_{\mu}$ whenever $\mu \sim \nu$, the proof of [14, Proposition 5.9] implies that for each $\mu \in I C(\Lambda)$, we have $\ell_{\mu}=l_{i}$ for some $i \in\{1, \ldots, n\}$. Consequently, $\max _{\mu \in I C(\Lambda)} \ell_{\mu}=$ $\max _{i=1}^{n} l_{i}$.

Conversely, suppose $C^{*}(\Lambda)$ is finite and $\max _{\mu \in I C(\Lambda)} \ell_{\mu}=1$. By [37, Lemma 5.1.2], $C^{*}(\Lambda)$ has no infinite projections. So [14, Corollary 5.7] implies that $C^{*}(\Lambda)$ is stably isomorphic to $\bigoplus_{i=1}^{n} C\left(\mathbb{T}^{l_{i}}\right)$ for some $n \geq 1$ and $l_{1}, \ldots, l_{n} \in\{0, \ldots, k\}$ such that $\max _{\mu \in I C(\Lambda)} \ell_{\mu}=\max _{i=1}^{n} l_{i}$. By the properties in Section 1.1, it follows that

$$
\operatorname{sr}\left(C^{*}(\Lambda)\right)=\operatorname{sr}\left(\bigoplus_{i=1}^{n} C\left(\mathbb{T}^{l_{i}}\right)\right)=\max _{i=1, \ldots, n}\left\lfloor l_{i} / 2\right\rfloor+1=1 .
$$

Remark 3.2. It turns out that $C^{*}$-algebras of finite $k$-graphs with $k>1$ rarely have stable rank one: the condition $\max _{\mu \in I C(\Lambda)} \ell_{\mu}=1$ is rather strict. As Proposition 3.3 indicates, if $\Lambda^{0}$ is finite and $\operatorname{sr}\left(C^{*}(\Lambda)\right)=1$ (hence stably finite), then any initial cycle in $\Lambda$ has at most one colour. Using Lemma 2.2(2) and the factorisation property, it follows that any cycle in $\Lambda$ has at most one colour.

Figure 5 illustrates two examples of 2-graphs $\Lambda$ with $C^{*}(\Lambda)$ of stable rank one. The first example, illustrated on the left, has two vertices $v_{1}, v_{2}$, a single edge red (dashed) loop based at $v_{1}$, and single edge blue (solid) loop based at $v_{2}$. The second example, shown on the right in Figure 5, is different in that it is connected and contains no loops.

Following [14], for $n \in \mathbb{N}^{k}$ there is a shift map $\sigma^{n}:\left\{x \in W_{\Lambda}: n \leq d(x)\right\} \rightarrow W_{\Lambda}$ such that $d\left(\sigma^{n}(x)\right)=d(x)-n$ and $\sigma^{n}(x)(p, q)=x(n+p, n+q)$ for $0 \leq p \leq q \leq$ $d(x)-n$ where we use the convention $\infty-a=\infty$ for $a \in \mathbb{N}$. For $x \in W_{\Lambda}$ and 
$n \leq d(x)$, we then have $x(0, n) \sigma^{n}(x)=x$. We now show an easy way to compute $\ell_{\mu}$, using only the degree of $\mu$.

Proposition 3.3. Let $\Lambda$ be a finite, locally convex $k$-graph such that $\Lambda$ has no cycle with an entrance. Then for each $\mu \in \operatorname{IC}(\Lambda)$,

$$
\ell_{\mu}=\left|\left\{i \leq k: d(\mu)_{i}>0\right\}\right| \mid \text {. }
$$

Proof. Let $I=\left\{i \leq k: d(\mu)_{i}>0\right\}$. We must show that $\ell_{\mu}=|I|$. If $I=\emptyset$ then $\ell_{\mu}=0=|I|$, so assume that $I$ is nonempty. By (1.1),

$$
G_{\mu}=\left\{m-n: n, m \leq d\left(\mu^{\infty}\right), \mu^{\infty}(m)=\mu^{\infty}(n)\right\} .
$$

Since each $n, m \leq d\left(\mu^{\infty}\right)$ satisfy $n, m \in \operatorname{span}_{\mathbb{N}}\left\{e_{i}: i \in I\right\}$, the rank of $G_{\mu}$ is at most $|I|$. Consequently, it suffices to show $G_{\mu}$ contains a subgroup of rank $|I|$.

Let $v:=\mu^{\infty}(0)$. We claim that for each colour $i \in I$, there exists a positive integer $m_{i}$ such that $\mu^{\infty}\left(0, m_{i} e_{i}\right)=v$. Indeed, since $\Lambda^{0}$ is finite there exists $m<n$ such that $\mu^{\infty}\left(m e_{i}\right)=\mu^{\infty}\left(n e_{i}\right)$. Now using that $\mu^{\infty} \in \Lambda^{\leq \infty}$ (see Lemma 2.1) and that for every vertex $w$ on $\mu$ there is a unique path in $w \Lambda^{\leq \infty}$ (see Section 1.7) we get that $\sigma^{m e_{i}}\left(\mu^{\infty}\right)=\sigma^{n e_{i}}\left(\mu^{\infty}\right)$. Now for $N:=m d(\mu)$ it follows that $\sigma^{N}\left(\mu^{\infty}\right)=\mu^{\infty}$. Since

$$
\mu^{\infty}=\sigma^{N}\left(\mu^{\infty}\right)=\sigma^{N-m e_{i}+m e_{i}}\left(\mu^{\infty}\right)=\sigma^{N-m e_{i}+n e_{i}}\left(\mu^{\infty}\right)=\sigma^{(n-m) e_{i}}\left(\mu^{\infty}\right),
$$

we get $\left(\mu^{\infty}\right)\left((n-m) e_{i}\right)=v$. Hence $\mu^{\infty}$ contains a cycle of degree $(n-m) e_{i}$ based at $v$. In particular we can use $m_{i}:=n-m$.

By the preceding paragraph $\left\{m_{i} e_{i}: i \in I\right\} \subseteq G_{\mu}$ is a $\mathbb{Z}$-linearly independent set generating a rank- $|I|$ subgroup of $G_{\mu}$. So the rank of $G_{\mu}$ is $|I|$.

Corollary 3.4. Let $\Lambda$ be a finite, locally convex $k$-graph. Then $\operatorname{sr}\left(C^{*}(\Lambda)\right)=1$ if and only if $\Lambda$ has no cycle with an entrance and no initial cycle with more than one colour.

Proof. Combine Proposition 2.7, Theorem 3.1, and Proposition 3.3.

Remark 3.5. A graph trace on a locally convex row-finite $k$-graph $\Lambda$ is a function $g: \Lambda^{0} \rightarrow \mathbb{R}^{+}$satisfying the graph trace property, $g(v)=\sum_{\lambda \in v \Lambda \leq n} g(s(\lambda))$ for all $v \in \Lambda^{0}$ and $n \in \mathbb{N}^{k}$. It is faithful if it is nonzero on every vertex in $\Lambda([27,45])$.

It can be shown that Corollary 3.4 remains valid if we replace "has no cycle with an entrance" by "admits a faithful graph trace". Indeed, the $C^{*}$-algebra of a row-finite and cofinal $k$-graph $\Lambda$ with no sources is stably finite if and only if $\Lambda$ admits a faithful graph trace [7, Theorem 1.1], and for $\Lambda^{0}$ finite this remains true without "cofinal" and with "locally convex" instead of "no sources" (by virtue of Theorem 2.5 and [28, Lemma 7.1]).

\section{Stable RAnK in the Simple And COfinal CASE.}

In this section we focus on stable rank of $k$-graph $C^{*}$-algebras for which the $k$ graph is cofinal, corresponding to boxes 1 and 3 in Figure 1 . Since simple $k$-graph $C^{*}$-algebras constitute a sub-case of this situation (as illustrated below), we consider those first.

Let $\Lambda$ be a row-finite, locally convex $k$-graph. Following [42], $\Lambda$ is cofinal if for all pairs $v, w \in \Lambda^{0}$ there exists $n \in \mathbb{N}^{k}$ such that $s\left(w \Lambda^{\leq n}\right) \subseteq s(v \Lambda)$. Following [35], $\Lambda$ has local periodicity $m, n$ at $v$ if for every $x \in v \Lambda \leq \infty$, we have $m-(m \wedge d(x))=$ 


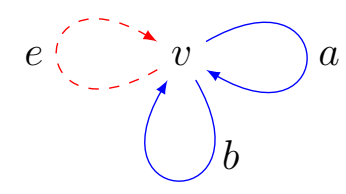

FiguRE 6. Example of 2-graph with a $C^{*}$-algebra of stable rank infinity.

$n-(n \wedge d(x))$ and $\sigma^{m \wedge d(x)}(x)=\sigma^{n \wedge d(x)}(x)$. If $\Lambda$ fails to have local periodicity $m, n$ at $v$ for all $m \neq n \in \mathbb{N}^{k}$ and $v \in \Lambda^{0}$, we say that $\Lambda$ has no local periodicity. By [35, Theorem 3.4],

$\Lambda$ is cofinal and has no local periodicity if and only if $C^{*}(\Lambda)$ is simple.

The stable rank of 1-graph $C^{*}$-algebras is well understood (see [9, Theorem 3.4], [18, Theorem 3.3] and [17, Theorem 3.1]), but the following is new for $k>1$. Recall that a cycle is a path $\lambda \in \Lambda \backslash \Lambda^{0}$ such that $r(\lambda)=s(\lambda)$.

Proposition 4.1. Let $\Lambda$ be a finite, locally convex $k$-graph. Suppose that $\Lambda$ is cofinal and has no local periodicity (i.e., $C^{*}(\Lambda)$ is simple). Then

$$
\operatorname{sr}\left(C^{*}(\Lambda)\right)= \begin{cases}1 & \text { if } \Lambda \text { contains no cycles } \\ \infty & \text { otherwise. }\end{cases}
$$

Proof. If $\Lambda$ contains no cycles then [14, Corollary 5.7] gives $C^{*}(\Lambda) \cong M_{\Lambda v}(\mathbb{C})$ for some vertex $v \in \Lambda^{0}$. Using [34, Proposition 1.7 and Theorem 3.6] we obtain that $\operatorname{sr}\left(C^{*}(\Lambda)\right)=1$.

If $\Lambda$ contains a cycle, then another application of [14, Corollary 5.7] (see also [3, Remark 5.8]) gives that $C^{*}(\Lambda)$ is purely infinite. Since $C^{*}(\Lambda)$ is unital, simple and purely infinite it contains two isometries with orthogonal ranges, so [34, Proposition 6.5] gives $\operatorname{sr}\left(C^{*}(\Lambda)\right)=\infty$.

In conclusion, the stable rank of a unital simple $k$-graph $C^{*}$-algebra is completely determined by presence or absence of a cycle in the $k$-graph.

4.1. The cofinal case. We now consider the cofinal case. We start by recalling a result of Jeong, Park and Shin about directed graphs (or 1-graphs). We refer to [18] for the terminology involved.

Proposition 4.2 ([18, Proposition 3.7]). Let $E$ be a locally finite directed graph. If $E$ is cofinal then either $\operatorname{sr}\left(C^{*}(E)\right)=1$ or $C^{*}(E)$ is purely infinite simple.

Remark 4.3. We illustrate why for $k$-graphs we can not hope for a result similar to Proposition 4.2. Consider the 2-graph $\Lambda$ in Figure 6 with two blue edges $a, b \in \Lambda^{e_{1}}$ and one red edge $e \in \Lambda^{e_{2}}$ and the factorisation property $a e=e a, b e=e b$. Since $\Lambda$ has only one vertex, it is automatically cofinal. However, $C^{*}(\Lambda)$ neither has stable rank one nor is purely infinite simple as the following discussion shows:

The $C^{*}$-algebra $C^{*}(\Lambda)$ fails to have stable rank one because it is not stably finite (containing a cycle with an entrance). It is not simple, so in particular not purely infinite simple because

$$
\text { for every } x \in v \Lambda^{\leq \infty} \text { we have } \sigma^{e_{2}}(x)=x,
$$


so $\Lambda$ has local periodicity $p=e_{2}, q=0$ at $v$ and $C^{*}(\Lambda)$ is non-simple.

Because of our particular choice of factorisation rules $a e=e a, b e=e b$ Lemma 1.7 implies that $C^{*}(\Lambda) \cong \mathcal{O}_{2} \otimes C(\mathbb{T})$. If we instead used the factorisation $a e=e b$, $b e=e a$, then Lemma 1.7 would not apply, but we would still have $\sigma^{2 e_{2}}(x)=x$ for each $x \in v \Lambda \leq \infty$ making $C^{*}(\Lambda)$ non-simple.

Remark 4.3 notwithstanding, we are able to provide a characterisation of stable rank in the cofinal case. Given a $C^{*}$-algebra $A$, we write $a \oplus b$ for the diagonal matrix $\operatorname{diag}(a, b)$ in $M_{2}(A)$ and write $\sim$ for the von Neumann equivalence relation between elements in matrix algebras over $A$. A unital $C^{*}$-algebra $A$ is properly infinite if $1 \oplus 1 \oplus r \sim 1$ for some projection $r$ in some matrix algebra over $A$ (for more details see [38]).

Theorem 4.4. Let $\Lambda$ be a cofinal, finite, locally convex $k$-graph. Suppose that $\Lambda$ contains a cycle with an entrance. Then $C^{*}(\Lambda)$ is properly infinite and has stable rank $\infty$.

Proof. Let $\mu$ be a cycle with an entrance $\tau$, that is

$$
\tau \in r(\mu) \Lambda, \quad d(\tau) \leq d\left(\mu^{\infty}\right), \quad \text { and } \quad \tau \neq \mu^{\infty}(0, d(\tau)) .
$$

Fix $n \geq 1$ such that $m:=d(\mu) n \geq d(\tau)$. Since $\tau \neq \mu^{\infty}(0, d(\tau))=\mu^{n}(0, d(\tau))$, there exists $\beta \in s(\tau) \Lambda$ such that $\mu^{n}$ and $\tau \beta$ are distinct elements of $r(\mu) \Lambda^{\leq m}$. Write $r(\mu) \Lambda^{\leq m}=\left\{\nu_{1}, \ldots, \nu_{N}\right\}$ with $\nu_{1}=\mu^{n}$ and $\nu_{2}=\tau \beta$. For each $i=1, \ldots, N$ set $v_{i}=s\left(\nu_{i}\right)$ and let $x=\left(s_{\nu_{1}}, \ldots, s_{\nu_{N}}\right)$. Then $x x^{*}=\sum_{\lambda \in v_{1} \Lambda \leq m} s_{\lambda} s_{\lambda}^{*}=s_{v_{1}}$. Moreover for $i \neq j, s_{\nu_{i}}^{*} s_{\nu_{j}}=0$, so

$$
s_{v_{1}}=x x^{*} \sim x^{*} x=\operatorname{diag}\left(s_{\nu_{1}}^{*} s_{\nu_{1}}, \ldots, s_{\nu_{N}}^{*} s_{\nu_{N}}\right)=s_{v_{1}} \oplus \cdots \oplus s_{v_{N}} .
$$

We claim that for any pair of vertices $u, v \in \Lambda^{0}$ there exist a constant $M_{u, v}$ and a projection $p_{u, v}$ in some matrix algebra over $C^{*}(\Lambda)$ such that

$$
\left(\bigoplus_{l=1}^{M_{u, v}} s_{u}\right) \sim s_{v} \oplus p_{u, v}
$$

To see this, fix $u, v \in \Lambda^{0}$. Since $\Lambda$ is cofinal there exists $n \in \mathbb{N}^{k}$ such that $s\left(v \Lambda^{\leq n}\right) \subseteq s(u \Lambda)$. Writing $v \Lambda^{\leq n}=\left\{\mu_{1}, \ldots, \mu_{M_{u, v}}\right\}$ and $u_{i}=s\left(\mu_{i}\right)$, we have $s_{v} \sim$ $s_{u_{1}} \oplus \cdots \oplus s_{u_{M_{u, v}}}$. Since $s\left(v \Lambda^{\leq n}\right)=\left\{u_{i}: i \leq M_{u, v}\right\} \subseteq s(u \Lambda)$, for each $i \leq M_{u, v}$ there exists $\lambda_{i} \in u \Lambda$ such that $s\left(\lambda_{i}\right)=u_{i}$. Let $m_{i}=d\left(\lambda_{i}\right)$ for each $i$. Then for each $i=1, \ldots, M_{u, v}$,

$$
s_{u}=\sum_{\lambda \in u \Lambda \leq m_{i}} s_{\lambda} s_{\lambda}^{*} \sim s_{u_{i}} \oplus p_{i}
$$

for some projection $p_{i}$ in a matrix algebra over $C^{*}(\Lambda)$. With $p_{u, v}=\bigoplus_{i} p_{i}$ we obtain

$$
\left(\bigoplus_{l=1}^{M_{u, v}} s_{u}\right) \sim\left(\bigoplus_{i=1}^{M_{u, v}} s_{u_{i}}\right) \oplus p_{u, v} \sim s_{v} \oplus p_{u, v},
$$

which establishes the claim.

Applying (4.2) to $u=v_{2}$ and $v=v_{1}$ we get

$$
\left(\bigoplus_{l=1}^{M_{v_{2}, v_{1}}} s_{v_{2}}\right) \sim s_{v_{1}} \oplus p_{v_{2}, v_{1}}
$$


Recall that $s_{v_{1}} \sim s_{v_{1}} \oplus s_{v_{2}} \oplus\left(\bigoplus_{i=3}^{N} s_{v_{i}}\right)$. Let $q:=p_{v_{2}, v_{1}} \oplus\left(\bigoplus_{l=1}^{M_{v_{2}, v_{1}}} \bigoplus_{i=3}^{N} s_{v_{i}}\right)$, meaning that if $N=2$ then $q=p_{v_{2}, v_{1}} \oplus 0$. Then

$$
s_{v_{1}} \sim s_{v_{1}} \oplus\left(\bigoplus_{l=1}^{M_{v_{2}, v_{1}}} s_{v_{2}}\right) \oplus\left(\bigoplus_{l=1}^{M_{v_{2}, v_{1}}} \bigoplus_{i=3}^{N} s_{v_{i}}\right) \sim s_{v_{1}} \oplus s_{v_{1}} \oplus q .
$$

Applying (4.2) to $u=v_{1}$ and to each $v \in \Lambda^{0} \backslash\left\{v_{1}\right\}$ at the second equality, and putting $L:=2+\sum_{v \in \Lambda^{0} \backslash\left\{v_{1}\right\}} M_{v_{1}, v}$, we calculate:

$$
\begin{aligned}
1 \oplus 1 \oplus\left(\bigoplus_{v \in \Lambda^{0} \backslash\left\{v_{1}\right\}} p_{v_{1}, v}\right) & \sim 1 \oplus s_{v_{1}} \oplus\left(\bigoplus_{v \in \Lambda^{0} \backslash\left\{v_{1}\right\}}\left(s_{v} \oplus p_{v_{1}, v}\right)\right) \\
& \sim 1 \oplus s_{v_{1}} \oplus\left(\bigoplus_{v \in \Lambda^{0} \backslash\left\{v_{1}\right\}}\left(\bigoplus_{i=1}^{M_{v_{1}, v}} s_{v_{1}}\right)\right) \\
& \sim\left(\bigoplus_{v \in \Lambda^{0} \backslash\left\{v_{1}\right\}} s_{v}\right) \oplus s_{v_{1}} \oplus s_{v_{1}} \oplus\left(\bigoplus_{v \in \Lambda^{0} \backslash\left\{v_{1}\right\}}\left(\bigoplus_{i=1}^{M_{v_{1}, v}} s_{v_{1}}\right)\right) \\
& \sim\left(\bigoplus_{v \in \Lambda^{0} \backslash\left\{v_{1}\right\}} s_{v}\right) \oplus\left(\bigoplus_{j=1}^{L} s_{v_{1}}\right) .
\end{aligned}
$$

Using (4.3) we have $s_{v_{1}} \sim s_{v_{1}} \oplus\left(\bigoplus_{j=1}^{L-1} s_{v_{1}}\right) \oplus\left(\bigoplus_{j=1}^{L-1} q\right)$, so $r=\left(\bigoplus_{v \in \Lambda^{0} \backslash\left\{v_{1}\right\}} p_{v_{1}, v}\right) \oplus$ $\left(\bigoplus_{j=1}^{L-1} q\right)$ satisfies

$$
1 \oplus 1 \oplus r \sim 1 .
$$

Hence 1 is properly infinite. Now [34, Proposition 6.5] gives $\operatorname{sr}\left(C^{*}(\Lambda)\right)=\infty$.

With Proposition 4.2 in mind, the following is a dichotomy for the $C^{*}$-algebras associated to cofinal finite $k$-graphs.

Corollary 4.5. Let $\Lambda$ be a cofinal, finite, locally convex $k$-graph. Then either $C^{*}(\Lambda)$ is stably finite and $\operatorname{sr}\left(C^{*}(\Lambda)\right)$ is given by Corollary 2.9, or $C^{*}(\Lambda)$ is properly infinite and $\operatorname{sr}\left(C^{*}(\Lambda)\right)=\infty$.

Proof. If $C^{*}(\Lambda)$ is not stably finite then $\Lambda$ contains a cycle with an entrance by Proposition 2.7. Hence $C^{*}(\Lambda)$ is properly infinite and $\operatorname{sr}\left(C^{*}(\Lambda)\right)=\infty$ by Theorem 4.4. Conversely, if $C^{*}(\Lambda)$ is properly infinite, then it is also infinite, so $C^{*}(\Lambda)$ is not finite and hence not stably finite. If $C^{*}(\Lambda)$ is stably finite then Corollary 2.9 applies.

Remark 4.6. Theorem 4.4 includes cases not covered by any of our preceding results. Consider for example the 2-graph $\Lambda$ in Figure 6. By Theorem 4.4 the associated $C^{*}$-algebra has stable rank infinity.

Example 4.7. By Corollary 4.5, we can compute the stable rank of $k$-graph $C^{*}$ algebras in boxes 1 to 3 in Figure 1. It therefore makes sense to consider the range of stable rank achieve by these $C^{*}$-algebra. In box 3 stable rank infinity can be obtained as in Remark 4.3. For finite stable rank, Table 1 lists a few $4 n$-graphs $\Lambda$ together with their associated $C^{*}$-algebra and its stable rank (we use a multiple of 4 because it makes the formulas for the stable rank simpler). 
TABLE 1. A few examples of $4 n$-graphs.

\begin{tabular}{|c|c|c|c|}
\hline $4 n$-graph $\Lambda$ & $|\Lambda \leq N|$ & $C^{*}(\Lambda)$ & $\operatorname{sr}\left(C^{*}(\Lambda)\right)$ \\
\hline$\left(v_{1}\right.$ & 1 & $C\left(\mathbb{T}^{4 n}\right)$ & $2 n+1$ \\
\hline$w_{1} \longleftarrow v_{1}$ & 2 & $M_{2}\left(C\left(\mathbb{T}^{4 n}\right)\right)$ & $n+1$ \\
\hline$w_{1} \underbrace{4 n}_{4 n}$ & 3 & $M_{3}\left(C\left(\mathbb{T}^{4 n}\right)\right)$ & $\left\lceil\frac{2 n}{3}\right\rceil+1$ \\
\hline 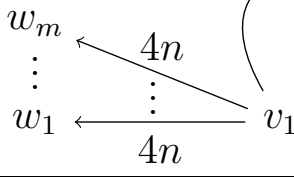 & $m+1$ & $M_{m+1}\left(C\left(\mathbb{T}^{4 n}\right)\right)$ & $\left\lceil\frac{2 n}{m+1}\right\rceil+1$ \\
\hline$v_{3 \overleftarrow{4 n}} v_{2 \overleftarrow{4 n}} v_{1}$ & $\left(\begin{array}{c}4 n \\
2\end{array}\right)$ & $M_{\left(\begin{array}{c}4 n \\
2\end{array}\right)}\left(C\left(\mathbb{T}^{4 n}\right)\right)$ & $\left|\frac{2 n}{\left(\begin{array}{c}4 n \\
2\end{array}\right)}\right|+1$ \\
\hline $\begin{array}{l}(\sqrt{2 n} \\
v_{2} \longleftarrow 2 n\end{array}$ & 2 & $M_{2}\left(C\left(\mathbb{T}^{4 n}\right)\right)$ & $n+1$ \\
\hline
\end{tabular}

Except for the last $4 n$-graph, each black edge represents exactly $4 n$ edges of different colours, one of each colour; the last $4 n$-graph has $2 n$ loops at $v_{2}$, one each of the first $2 n$ colours and $2 n$ edges from $v_{1}$ to $v_{2}$, one each of the remaining $2 n$ colours. Each example admits a unique factorisation rule, so each illustration in Table 1 represents a unique $4 n$-graph.

\section{Stable RANK In THE NON-STABly Finite, NON-COFinAl CASE}

So far we have looked at the stably finite case (including stable rank one) and the cofinal case (including the simple case). Here we study the remaining case corresponding to box 4 in Figure 1.

We start by revisiting the cofinality condition for row-finite locally convex $k$ graphs. Following [35], a subset $H \subseteq \Lambda^{0}$ is hereditary if $s(H \Lambda) \subseteq H$. We say $H$ is 
saturated if for all $v \in \Lambda^{0}$,

$$
\left\{s(\lambda): \lambda \in v \Lambda^{\leq e_{i}}\right\} \subseteq H \text { for some } i \in\{1, \ldots, k\} \Longrightarrow v \in H .
$$

or equivalently, if $v \notin H$ implies that for each $n \in \mathbb{N}^{k}, s\left(v \Lambda^{\leq n}\right) \nsubseteq \subseteq H$ (see Lemma 5.1). The relevant characterisation of cofinal is included in Lemma 5.2 below with a short proof based on [23] and [42]. Since this paper focuses on unital $k$-graph $C^{*}$-algebras, it is worth pointing out that Lemmas 5.1 and 5.2 do not assume that $\left|\Lambda^{0}\right|<\infty$.

Lemma 5.1. Let $\Lambda$ be a row-finite locally convex $k$-graph. Then $H \subseteq \Lambda^{0}$ is saturated if and only if for all $v \in \Lambda^{0}, v \notin H$ implies that for each $n \in \mathbb{N}^{k}, s\left(v \Lambda^{\leq n}\right) \nsubseteq H$.

Proof. Fix $v \in \Lambda^{0}$. Suppose $v \notin H$. Since $\Lambda$ is saturated, for all $i \leq k,\{s(\lambda)$ : $\left.\lambda \in v \Lambda^{\leq e_{i}}\right\} \nsubseteq H$. Clearly $s\left(v \Lambda^{\leq m}\right) \nsubseteq H$ for $m=0$. Fix any $m \in \mathbb{N}^{k} \backslash\{0\}$. Set $\left(n^{(0)}, v^{(0)}, \lambda^{(0)}\right)=(m, v, v)$. Choose $i$ such that $n_{i}^{(0)} \neq 0$. Since $v^{(0)} \notin H$, there exists $\mu^{(1)} \in v^{(0)} \Lambda \leq e_{i} \backslash \Lambda H$. Set $\left(n^{(1)}, v^{(1)}, \lambda^{(1)}\right)=\left(n^{(0)}-e_{i}, s\left(\mu^{(1)}\right), \lambda^{(0)} \mu^{(1)}\right)$. Choose $i$ such that $n_{i}^{(1)} \neq 0$. Since $v^{(1)} \notin H$, there exists $\mu^{(2)} \in v^{(1)} \Lambda \leq e_{i} \backslash \Lambda H$. Set $\left(n^{(2)}, v^{(2)}, \lambda^{(2)}\right)=\left(n^{(1)}-e_{i}, s\left(\mu^{(2)}\right), \lambda^{(1)} \mu^{(2)}\right)$.

For each step, $\left|n^{(i)}\right|=|m|-i$, so $l=|m|$ satisfies $n^{(l)}=0$. Notice that $\lambda^{(0)} \in v \Lambda^{\leq\left(m-n^{(0)}\right)}, \lambda^{(1)} \in v \Lambda^{\leq\left(m-n^{(1)}\right)}, \ldots, \lambda^{(l)} \in v \Lambda^{\leq\left(m-n^{(l)}\right)}$. Hence $\lambda^{(l)} \in v \Lambda^{\leq m}$ and $s\left(\lambda^{(l)}\right) \notin H$ so $s(v \Lambda \leq m) \nsubseteq H$.

Lemma 5.2 ([23, 42]). Let $\Lambda$ be a row-finite locally convex $k$-graph. Then the following are equivalent:

(1) $\Lambda$ is cofinal;

(2) for all $v \in \Lambda^{0}$, and $\left(\lambda_{i}\right)$ with $\lambda_{i} \in \Lambda^{\leq(1, \ldots, 1)}$, and $s\left(\lambda_{i}\right)=r\left(\lambda_{i+1}\right)$ there exist $i \in \mathbb{N}$ and $n \leq d\left(\lambda_{i}\right)$ such that $v \Lambda \lambda_{i}(n) \neq \emptyset$; and

(3) $\Lambda^{0}$ contains no nontrivial hereditary saturated subsets.

Proof. Firstly we show that $(1) \Rightarrow(3)$. Suppose (1) and suppose that $H \subseteq \Lambda^{0}$ is a nonempty hereditary, saturated set. We show that $H=\Lambda^{0}$. Fix $v \in \Lambda^{0}$. Since $H$ is nonempty, there exists $w \in H$. By (1) there exists $n \in \mathbb{N}^{k}$ such that $s\left(v \Lambda^{\leq n}\right) \subseteq$ $s(w \Lambda)$. Since $H$ is hereditary, $s\left(v \Lambda^{\leq n}\right) \subseteq s(H \Lambda) \subseteq H$. Hence Lemma 5.1 gives $v \in H$.

Now we show that $(3) \Rightarrow(2)$. Suppose that (2) fails, that is, there exist $v \in \Lambda^{0}$, and a sequence $\left(\lambda_{i}\right)$ with $\lambda_{i} \in \Lambda^{\leq(1, \ldots, 1)}, s\left(\lambda_{i}\right)=r\left(\lambda_{i+1}\right)$ for all $i$ such that for all $i \in \mathbb{N}$ and all $n \leq d\left(\lambda_{i}\right)$ we have $v \Lambda \lambda_{i}(n)=\emptyset$. Let

$$
H=\left\{w \in \Lambda^{0}: w \Lambda \lambda_{i}(n)=\emptyset \text { for all } i \in \mathbb{N} \text { and } n \leq d\left(\lambda_{i}\right)\right\} .
$$

Then $H$ is nontrivial as $v \in H$ and hereditary because if $u \Lambda w \neq \emptyset$ then $s(w \Lambda) \subseteq$ $s(u \Lambda)$. To show that $H$ is saturated take $u \in \Lambda^{0}$ and $j \leq k$ such that $s\left(u \Lambda \leq e_{j}\right) \subseteq$ $H$. We must show that $u \in H$. Assume otherwise for contradiction. We have $u \notin s\left(u \Lambda^{\leq e_{j}}\right)$ because otherwise $u=s(u)$ belongs to $H$, so $u \Lambda^{e_{j}} \neq \emptyset$. Since $u \notin H$, there exists $\lambda \in u \Lambda$ such that $s(\lambda)=\lambda_{i}(n)$ for some $i, n$. We claim that $d(\lambda)_{j}=0$. Indeed, if not, then $\lambda=\mu \mu^{\prime}$ for some $\mu \in u \Lambda^{e_{j}}$. We then have $s(\mu) \in s\left(u \Lambda^{e_{j}}\right) \subseteq H$, so $s(\mu) \Lambda \lambda_{i}(n)=\emptyset$ contradicting $\mu^{\prime} \in s(\mu) \Lambda \lambda_{i}(n)$. Since $\Lambda$ is locally convex and $d(\lambda)_{j}=0$ and since $u \Lambda^{e_{j}} \neq \emptyset$, we have $\lambda_{i}(n) \Lambda^{e_{j}}=s(\lambda) \Lambda^{e_{j}} \neq \emptyset$. Let $\beta=\lambda_{i}\left(n, d\left(\lambda_{i}\right)\right)$. Since $\Lambda$ is locally convex, either $d(\beta)_{j} \neq 0$ or $s\left(\lambda_{i}\right) \Lambda^{e_{j}} \neq \emptyset$. Since $\lambda_{i+1} \in \Lambda \leq(1, \ldots, 1)$ it follows that $d\left(\beta \lambda_{i+1}\right) \geq e_{j}$. Now $\lambda^{\prime}:=\lambda \beta \lambda_{i+1} \in u \Lambda$ satisfies $s\left(\lambda^{\prime}\right)=\lambda_{i^{\prime}}\left(n^{\prime}\right)$ for some 


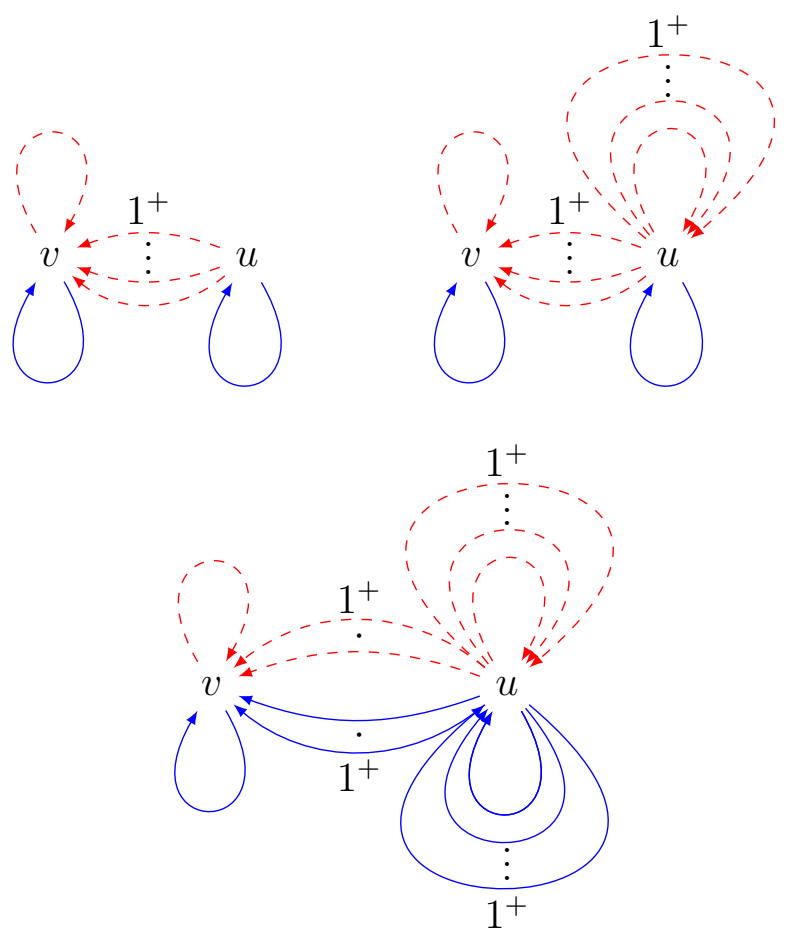

Figure 7. Example of 2-graphs $\Lambda$ with $C^{*}(\Lambda)$ of stable rank two or thee.

$i^{\prime}, n^{\prime}$. But then, just as we got $d(\lambda)_{j}=0$, we deduce $d\left(\lambda^{\prime}\right)_{j}=0$, a contradiction. So $H$ is saturated, so (3) does not hold.

Finally we prove $(2) \Rightarrow(1)$. Given (2), we suppose that (1) fails, and we derive a contradiction. Since (1) fails, there exist $v, w \in \Lambda^{0}$ such that for all $n \in \mathbb{N}^{k}$, we have $s\left(w \Lambda^{\leq n}\right) \nsubseteq s(v \Lambda)$. Set

$$
K=\left\{u \in \Lambda^{0}: s\left(u \Lambda^{\leq n}\right) \nsubseteq s(v \Lambda) \text { for all } n \in \mathbb{N}^{k}\right\} .
$$

Fix $u \in K$ and $j \leq k$. We claim that there exists $\mu \in u \Lambda^{\leq e_{j}}$ such that $s(\mu) \in K$. Indeed if $s\left(u \Lambda^{\leq e_{j}}\right) \subseteq \Lambda^{0} \backslash K$, then for each $\mu \in u \Lambda^{\leq e_{j}}$ there exists $n_{\mu} \in \mathbb{N}^{k}$ such that $s\left(\mu \Lambda^{n_{\mu}}\right) \subseteq s(v \Lambda)$. Since $s(v \Lambda)$ is hereditary, it follows that $n=\bigvee_{\mu \in u \Lambda^{\leq e_{j}}} n_{\mu}$ satisfies $s\left(u \Lambda^{\leq n+e_{j}}\right)=\bigcup_{\mu \in u \Lambda^{\leq e_{j}}} s\left(\mu \Lambda^{\leq n}\right) \subseteq s(v \Lambda)$, contradicting $u \in K$.

Since $w \in K$ we can construct a sequence $\left(\lambda_{i}\right)$ such that each $\lambda_{i} \in \Lambda \leq(1, \ldots, 1)$, each $s\left(\lambda_{i}\right)=r\left(\lambda_{i+1}\right)$, and for each $n \leq d\left(\lambda_{i}\right)$ we have $\lambda_{i}(n) \in K$. By (2) there exist $i$ and $n \leq d\left(\lambda_{i}\right)$ such that $v \Lambda \lambda_{i}(n) \neq \emptyset$, i.e., such that $s\left(\lambda_{i}(n) \Lambda^{\leq 0}\right) \subseteq s(v \Lambda)$. So $\lambda_{i}(n) \notin K$, a contradiction.

Remark 5.3. When a $k$-graph $\Lambda$ has only one vertex, it is automatically cofinal, and we deduce that the stable rank of $C^{*}(\Lambda)$ is infinite if there exists $j \leq k$ such that $\left|\Lambda^{e_{j}}\right| \geq 2$, and is equal to $\lfloor k / 2\rfloor+1$ if each $\left|\Lambda^{e_{j}}\right|=1$.

Remark 5.4. We now present all the 2-graphs $\Lambda$ with $\left|\Lambda^{0}\right|=2$ for which we have been unable to compute the stable rank of the associated $C^{*}$-algebra $C^{*}(\Lambda)$ (see Figure 7). In each case the 2-graph $\Lambda$ fails to be cofinal, because $\Lambda^{0}$ contains one nontrivial hereditary saturated subset, denoted $H$. 
In Figure 7, for each 2-graph $\Lambda$ the $C^{*}$-algebra $C^{*}(\Lambda)$ is non-simple with $H=\{u\}$. In the first case, we have $C^{*}(H \Lambda) \cong C(\mathbb{T})$, which has stable rank 1 , and so $I_{H}$ has stable rank 1 because stable rank 1 is preserved by stable isomorphism. In the remaining two cases, if there is one loop of each colour at $u$ then $C^{*}(H \Lambda) \cong C\left(\mathbb{T}^{2}\right)$ has stable rank 2, and otherwise, Theorem 4.4 implies that $C^{*}(H \Lambda)$ has stable rank $\infty$; either way, since $I_{H} \cong C^{*}(H \Lambda) \otimes \mathcal{K}^{\mathbb{I}}$, we have $\operatorname{sr}\left(I_{H}\right)=2$ as discussed in Section 1.1.

In all three cases, the quotient of $C^{*}(\Lambda)$ by $I_{H}$ is $C^{*}(\Lambda) / I_{H} \cong C^{*}(\Lambda \backslash \Lambda H) \cong C\left(\mathbb{T}^{2}\right)$. Hence, by [2, V.3.1.21] we deduce that $\operatorname{sr}\left(C^{*}(\Lambda)\right) \in\{2,3\}$, but we have been unable to determine the exact value in any of these cases.

Perhaps the easiest-looking case is the 2-graph (top left) with one red (dashed) edge from $u$ to $v$. In this case $C^{*}(\Lambda) \cong \mathcal{T} \otimes C(\mathbb{T})$, where $\mathcal{T}$ is the Toeplitz algebra generated by the unilateral shift. Despite knowing the stable rank of each of the components $(\operatorname{sr}(\mathcal{T})=2$ and $\operatorname{sr}(C(\mathbb{T}))=1)$ the stable rank of the tensor product is not known (there is no general formula for stable rank of tensor products).

\section{REFERENCES}

[1] A. an Huef and I. Raeburn. The ideal structure of Cuntz-Krieger algebras. Ergodic Theory Dynam. Systems, 17(3):611-624, 1997.

[2] B. Blackadar. Operator algebras, volume 122 of Encyclopaedia of Mathematical Sciences. Springer-Verlag, Berlin, 2006. Theory of $C^{*}$-algebras and von Neumann algebras, Operator Algebras and Non-commutative Geometry, III.

[3] J.H. Brown, L.O. Clark, and A. an Huef. Dense subalgebras of purely infinite simple groupoid C*-algebras. Proc. Edinb. Math. Soc. (2), 63(3):609-629, 2020.

[4] L.G. Brown. On higher real and stable ranks for $C C R C^{*}$-algebras. Trans. Amer. Math. Soc., 368(10):7461-7475, 2016.

[5] T.M. Carlsen, S. Kang, J. Shotwell, and A. Sims. The primitive ideals of the Cuntz-Krieger algebra of a row-finite higher-rank graph with no sources. J. Funct. Anal., 266(4):2570-2589, 2014.

[6] J. Castillejos, S. Evington, A. Tikuisis, S. White, and W. Winter. Nuclear dimension of simple $C^{*}$-algebras. Invent. Math., 224:245-290, 2021.

[7] L.O. Clark, A. an Huef, and A. Sims. AF-embeddability of 2-graph algebras and quasidiagonality of $k$-graph algebras. J. Funct. Anal., 271(4):958-991, 2016.

[8] K.R. Davidson and D. Yang. Representations of higher rank graph algebras. New York J. Math., 15:169-198, 2009.

[9] K. Deicke, J.H. Hong, and W. Szymański. Stable rank of graph algebras. Type I graph algebras and their limits. Indiana Univ. Math. J., 52(4):963-979, 2003.

[10] M. Dădărlat, G. Nagy, A. Némethi, and C. Pasnicu. Reduction of topological stable rank in inductive limits of $C^{*}$-algebras. Pacific J. Math., 153(2):267-276, 1992.

[11] S. Eilers and G.A. Elliott. The Riesz property for the $K_{*}$-group of a $C^{*}$-algebra of minimal stable and real rank. C. R. Math. Acad. Sci. Soc. R. Can., 25(4):108-113, 2003.

[12] G.A. Elliott, T.M. Ho, and A.S. Toms. A class of simple $C^{*}$-algebras with stable rank one. J. Funct. Anal., 256(2):307-322, 2009.

[13] G.A. Elliott and Z. Niu. On the classification of simple amenable $C^{*}$-algebras with finite decomposition rank. In Operator algebras and their applications, volume 671 of Contemp. Math., pages 117-125. Amer. Math. Soc., Providence, RI, 2016.

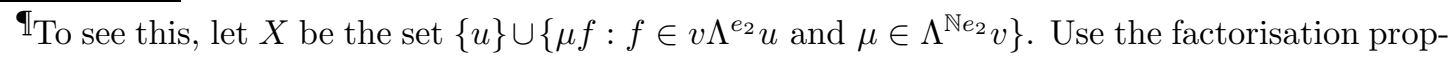
erty and the Cuntz-Krieger relations to see that $I_{H}=\overline{\operatorname{span}}\left\{s_{\mu} a s_{\nu}^{*}: \mu, \nu \in X\right.$ and $\left.a \in s_{u} C^{*}(\Lambda) s_{u}\right\}$. It is routine that for any finite subset $F \subseteq X$, the set $\left\{s_{\mu} s_{\nu}^{*}: \mu, \nu \in F\right\}$ is a system of matrix units. So Lemma 2.4 gives $\overline{\operatorname{span}}\left\{s_{\mu} a s_{\nu}^{*}: \mu, \nu \in F\right.$ and $\left.a \in s_{u} C^{*}(\Lambda) s_{u}\right\} \cong s_{u} C^{*}(\Lambda) s_{u} \otimes M_{|F|}(\mathbb{C})$. Taking the direct limit gives $I_{H} \cong s_{u} C^{*}(\Lambda) s_{u} \otimes \mathcal{K}\left(\ell^{2}(X)\right)$. 
[14] D.G. Evans and A. Sims. When is the Cuntz-Krieger algebra of a higher-rank graph approximately finite-dimensional? J. Funct. Anal., 263(1):183-215, 2012.

[15] I. Farah and M. Rørdam. Axiomatizability of the stable rank of $\mathrm{C}^{*}$-algebras. Münster J. Math., 10(2):269-275, 2017.

[16] R. Hazlewood, I. Raeburn, A. Sims, and S.B.G. Webster. Remarks on some fundamental results about higher-rank graphs and their $C^{*}$-algebras. Proc. Edinb. Math. Soc. (2), 56(2):575$597,2013$.

[17] J.A. Jeong. Stable rank and real rank of graph $C^{*}$-algebras. In Operator algebras and applications, volume 38 of Adv. Stud. Pure Math., pages 97-106. Math. Soc. Japan, Tokyo, 2004.

[18] J.A. Jeong, G.H. Park, and D.Y. Shin. Stable rank and real rank of graph $C^{*}$-algebras. Pacific J. Math., 200(2):331-343, 2001.

[19] E. Kirchberg. Exact $\mathrm{C}^{*}$-algebras, tensor products, and the classification of purely infinite algebras. In Proceedings of the International Congress of Mathematicians, Vol. 1, 2 (Zürich, 1994), pages 943-954. Birkhäuser, Basel, 1995.

[20] E. Kirchberg and M. Rørdam. Non-simple purely infinite $C^{*}$-algebras. Amer. J. Math., 122(3):637-666, 2000.

[21] A. Kumjian and D. Pask. Higher rank graph $C^{*}$-algebras. New York J. Math., 6:1-20, 2000.

[22] A. Kumjian, D. Pask, and A. Sims. $C^{*}$-algebras associated to coverings of $k$-graphs. Doc. Math., 13:161-205, 2008.

[23] P. Lewin and A. Sims. Aperiodicity and cofinality for finitely aligned higher-rank graphs. Math. Proc. Cambridge Philos. Soc., 149(2):333-350, 2010.

[24] H. Osaka and N.C. Phillips. Stable and real rank for crossed products by automorphisms with the tracial Rokhlin property. Ergodic Theory Dynam. Systems, 26(5):1579-1621, 2006.

[25] D. Pask, J. Quigg, and I. Raeburn. Coverings of $k$-graphs. J. Algebra, 289(1):161-191, 2005.

[26] D. Pask, I. Raeburn, M. Rørdam, and A. Sims. Rank-two graphs whose $C^{*}$-algebras are direct limits of circle algebras. J. Funct. Anal., 239(1):137-178, 2006.

[27] D. Pask, A. Rennie, and A. Sims. The noncommutative geometry of $k$-graph $C^{*}$-algebras. $J$. K-Theory, 1(2):259-304, 2008.

[28] D. Pask, A. Sierakowski, and A. Sims. Twisted $k$-graph algebras associated to Bratteli diagrams. Integral Equations Operator Theory, 81(3):375-408, 2015.

[29] N.C. Phillips. A classification theorem for nuclear purely infinite simple $C^{*}$-algebras. Doc. Math., 5:49-114, 2000.

[30] I.F. Putnam. On the topological stable rank of certain transformation group $C^{*}$-algebras. Ergodic Theory Dynam. Systems, 10(1):197-207, 1990.

[31] I. Raeburn and A. Sims. Product systems of graphs and the Toeplitz algebras of higher-rank graphs. J. Operator Theory, 53(2):399-429, 2005.

[32] I. Raeburn, A. Sims, and T. Yeend. Higher-rank graphs and their $C^{*}$-algebras. Proc. Edinb. Math. Soc. (2), 46(1):99-115, 2003.

[33] N. Riedel. On the topological stable rank of irrational rotation algebras. J. Operator Theory, 13(1):143-150, 1985.

[34] M.A. Rieffel. Dimension and stable rank in the $K$-theory of $C^{*}$-algebras. Proc. London Math. Soc. (3), 46(2):301-333, 1983.

[35] D. Robertson and A. Sims. Simplicity of $C^{*}$-algebras associated to row-finite locally convex higher-rank graphs. Israel J. Math., 172:171-192, 2009.

[36] M. Rørdam. The stable and the real rank of $\mathcal{Z}$-absorbing $C^{*}$-algebras. Internat. J. Math., 15(10):1065-1084, 2004.

[37] M. Rørdam, F. Larsen, and N. Laustsen. An introduction to $K$-theory for $C^{*}$-algebras, volume 49 of London Mathematical Society Student Texts. Cambridge University Press, Cambridge, 2000.

[38] M. Rørdam and E. Størmer. Classification of nuclear $C^{*}$-algebras. Entropy in operator algebras, volume 126 of Encyclopaedia of Mathematical Sciences. Springer-Verlag, Berlin, 2002. Operator Algebras and Non-commutative Geometry, 7.

[39] E. Ruiz, A. Sims, and A.P.W. Sørensen. UCT-Kirchberg algebras have nuclear dimension one. Adv. Math., 279:1-28, 2015. 
[40] Y. Sato, S. White, and W. Winter. Nuclear dimension and $\mathcal{Z}$-stability. Invent. Math., 202(2):893-921, 2015.

[41] C.P. Schafhauser. Finiteness properties of certain topological graph algebras. Bull. Lond. Math. Soc., 47(3):443-454, 2015.

[42] A. Sims. Gauge-invariant ideals in the $C^{*}$-algebras of finitely aligned higher-rank graphs. Canad. J. Math., 58(6):1268-1290, 2006.

[43] A. Sims and D.P. Williams. Amenability for Fell bundles over groupoids. Illinois J. Math., 57(2):429-444, 2013.

[44] Y. Suzuki. Almost Finiteness for General Étale Groupoids and Its Applications to Stable Rank of Crossed Products. Int. Math. Res. Not. IMRN, 19:6007-6041, 2020.

[45] M. Tomforde. The ordered $K_{0}$-group of a graph $C^{*}$-algebra. C. R. Math. Acad. Sci. Soc. R. Can., 25(1):19-25, 2003.

[46] J. Villadsen. On the stable rank of simple $C^{*}$-algebras. J. Amer. Math. Soc., 12(4):1091-1102, 1999.

Email address: dpask, asierako, asims@uow.edu.au

School of Mathematics and Applied Statistics, University of Wollongong, WolLONGONG NSW 2522, AUSTRALIA 